University of Wollongong

Research Online

Faculty of Engineering and Information

Faculty of Engineering and Information

Sciences - Papers: Part A

Sciences

$1-1-2012$

Self-mixing interferometry used for instrumentations and measurements(invited)

Silvano Donati

University of Pavia

Zhao Wang

Xi'an Jiaotong University

Yanguang Yu

University of Wollongong, yanguang@uow.edu.au

Follow this and additional works at: https://ro.uow.edu.au/eispapers

Part of the Engineering Commons, and the Science and Technology Studies Commons

Research Online is the open access institutional repository for the University of Wollongong. For further information contact the UOW Library: research-pubs@uow.edu.au 


\title{
Self-mixing interferometry used for instrumentations and measurements(invited)
}

\begin{abstract}
This paper reviews the development of a new configuration of interferometry, Self-mixing Interferometry(SMI). SMI is attractive because it doesn't require any optical part external to the laser and can be employed in a variety of measurements, ranging from the traditional measurements related to optical path length like displacements, small-amplitude vibrations, velocity; to sensing of weak optical echoes, like return loss and isolation factor measurements; and also to a special feature for the interaction with the medium, measurements of physical parameters, like laser line width, coherence length, and the alfa factor. Because it is also a coherent detection scheme, the SMI operation is close to the quantum limit of received field and the minimum detectable amplitudes of $20 \mathrm{pm} / \mathrm{V} \mathrm{Hz}$ or better are currently achieved upon operation on diffusive targets, whereas a corner cube allows half-wavelength counting mode or $0.5 \mu \mathrm{m}$ resolution on a dynamic range up to $2 \mathrm{~m}$. SMI has a compact setup, and is easy to deploy in the field and can interface a variety of experiments from MEMS testing to rotating machine vibration testing to pickup of biological motility as described in the paper.
\end{abstract}

\section{Keywords}

instrumentations, measurements, self, invited, used, interferometry, mixing

Disciplines

Engineering | Science and Technology Studies

\section{Publication Details}

Donati, S., Wang, Z. \& Yu, Y. (2012). Self-mixing interferometry used for instrumentations and measurements(invited). Chinese Optics, 5 (2), 93-115. 


\title{
用于光电仪器和相关测量的自混合干涉技术 (特邀)
}

\author{
DONATI Silvano ${ }^{*}$, 王 昭 ${ }^{2}$, 禹延光 ${ }^{3}$ \\ (1. 意大利帕维亚大学电子系, 伦巴第大区帕维亚 27100 , 意大利; \\ 2. 西安交通大学机械工程学院, 陕西西安 710049 ;
}

3. 澳大利亚伍伦贡大学电子、计算机及通讯工程学院, 新南威尔士州伍伦贡 2522 , 澳大利亚)

\begin{abstract}
摘要: 回顾了一种全新结构的干涉仪一自混合干涉仪 (SMI) 的发展情况。SMI 由于其在激光器外部无需任何光学元件且 应用范围广泛而倍受关注。SMI 可用于测量与光路长度有关的一些量 (如位移、小幅度振动、速度), 也可用于测量弱光 的回波 (即回波损耗和隔离因子的测量) 并可表征与介质相互作用特性相关的物理参数 (如激光线宽、相干长度以及 $\alpha$ 因子等)。SMI 是一种相干探测方法, 其工作在靠近接收场的量子极限处, 目前对散射目标物的最小探测幅值可达 $20 \mathrm{pm} / \sqrt{\mathrm{Hz}}$ 甚至更高, 使用角雉棱镜使其计数步长为半波长, 在动态范围为 $2 \mathrm{~m}$ 时其分辨率约为 $0.5 \mu \mathrm{m}$ 。另外, SMI 结 构紧凑、易于现场安装且可用于 MEMS 测试、旋转机械振动测试和生物运行测试等各种实验。
\end{abstract}

关 键 词: 自混合千涉术; 光学仪器;曲线拟合;激光二极管

中图分类号:TH744.3;0436.1 文献标识码:A doi:10.3788/CO.20120502.0093

\section{Self-mixing interferometry used for instrumentations and measurements (invited)}

DONATI Silvano $^{1 *}$, WANG Zhao ${ }^{2}$, YU Yan-guang ${ }^{3}$

(1. Department of Electronics, University of Pavia, Pavia 27100, Italy;

2. School of Mechanical Engineering, Xi'an Jiaotong University, Xi'an 710049, China;

3. School of Electrical, Computer and Telecommunications Engineering,

University of Wollongong, Wollongong 2522 ,Australia)

* Corresponding author, E-mail:silvano.donati@ unipv.it

\begin{abstract}
This paper reviews the development of a new configuration of interferometry, Self-mixing Interferometry ( SMI). SMI is attractive because it doesn't require any optical part external to the laser and can be employed in a variety of measurements, ranging from the traditional measurements related to optical path length like displacements, small-amplitude vibrations, velocity; to sensing of weak optical echoes, like return loss and isolation factor measurements; and also to a special feature for the interaction with the medium, measurements of physical parameters, like laser line width, coherence length, and the alfa factor. Because it is also a
\end{abstract}

收稿日期: 2011-12-15; 修订日期:2012-01-13 
coherent detection scheme, the SMI operation is close to the quantum limit of received field and the minimum detectable amplitudes of $20 \mathrm{pm} / \sqrt{\mathrm{Hz}}$ or better are currently achieved upon operation on diffusive targets, whereas a corner cube allows half-wavelength counting mode or $0.5 \mu \mathrm{m}$ resolution on a dynamic range up to $2 \mathrm{~m}$. SMI has a compact setup, and is easy to deploy in the field and can interface a variety of experiments from MEMS testing to rotating machine vibration testing to pickup of biological motility as described in the paper.

Key words: self-mixing interferometry; optical instruments; curve fitting;laser diodes

\section{1 引 言}

激光自混合干涉 (SMI) 技术起源于人们对激 光器中反馈光的研究。由于激光器发出的光被外 部物体反射或散射后回到激光器谐振器内时, 会 与内部腔场相互作用, 从而对激光器本身的性能 产生严重影响。因此, 人们起初研究的目的是要 消除这些影响。不过, 随着研究的深人, 人们发现 了反馈光对激光器功率的影响规律, 这一规律形 成了现在的 SMI 理论, 并将其应用于位移、距离、 振动、速度等不同参数的测量。由于 SMI 仪器与 传统的干涉仪具有同等的灵敏度,但其结构简单 紧凑、易于集成并且成本低、适用范围宽，所以在 很多场合都可以取代传统干涉仪。

\section{SMI 千涉仪工作原理}

SMI 干涉仪的结构不同于其它采用激光作光 源的光学干涉仪, 它是利用腔场与目标散射返回 场之间的相互作用，即耦合现象的一种特殊情况 来形成单光束自混合干涉的。由于干涉信号携有 被照射物体的运动信息, 故可用于振动、位移等参 数的测量。从激光器的早期研究开始, 人们就很 关注耦合现象的研究。1972 年, 诺贝尔奖获得者 H. Lamb Jr. 和 M. B. Spencer 两人发表了关于耦合 现象的论文, 论文对三反射镜激光器 (或自注人 式 $)^{[1]}$ 和互注人式 (双光源) 情况 ${ }^{[2]}$ 进行了描述, 这对后期耦合系统的发展起到了重要作用。

基于耦合方式的系统可分为互耦合系统 (两 个激光器相互改变它们各自的出射状态) 和自耦 合系统 (一个激光器接受从远距离目标返回的信 号)。耦合强度分为弱耦合和强耦合, 在激光腔
中产生的扰动从 $10^{-3}$ 下降到 $10^{-8}$ 为弱耦合 (SMI 情况); 现存的场功率上升到 $10^{-2}$ 为强耦合 (即产 生混沌)。在自耦合弱区, 振荡场的振幅调制 $(\mathrm{AM})$ 和频率调制 $(\mathrm{FM})$ 随变量 (或调制量) 正比 于返回场, 即正比于被扰动激光器的外部光学相 移 $2 k s$ ( $\boldsymbol{k}$ 为波矢, $s$ 为距离) 的正弦或余弦, SMI 的 应用正是利用了这一机理。自混合调制可以用旋 转矢量叠加的结果来解释 ${ }^{[3]}$, 如图 1 所示。

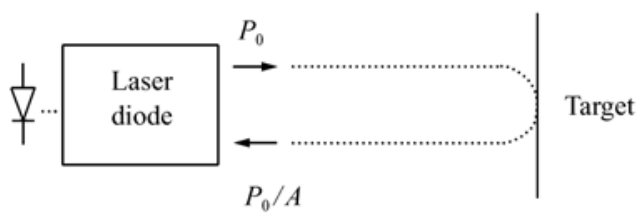

(a)

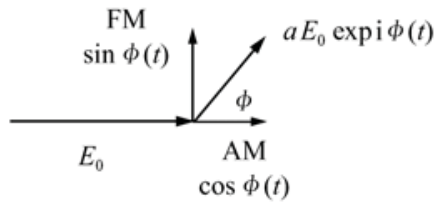

(b)

图 1 自混合干涉仪的基本结构 (a) 和旋转矢量叠加 模型 (b), 模型解释了 AM 和 FM 的产生原理, 它们分别是同相 $(\cos \phi)$ 和正交的 $(\sin \phi)$ 分量, $\phi=2 \boldsymbol{k} s$ 是到远距离目标并返回光路的光学相 移

Fig. 1 Basic scheme of a self-mixing interferometer (a). The rotating-vector addition model (b)
explains how AM and FM modulations are gen-
erated, respectively by the in-phase $(\cos \phi)$
the in-quadrature $(\sin \phi)$ components. $\phi=2 \boldsymbol{k} s$
is the optical phase shift of path to the remote
target and back

设 $E_{0}$ 是未受干扰的腔场, 则 $a E_{0}$ expi $2 \boldsymbol{k s}$ 为从 目标返回的场, $a$ 是传输过程衰减因子, $2 \boldsymbol{k} s$ 是相 位延迟。由通讯理论可知, 旋转矢量的叠加将产 
生一个由调制项同相位分量驱动的一个 $\operatorname{AM}\left(a E_{0}\right.$ $\cos 2 \boldsymbol{k} s)$ 和由正交分量驱动的 $\mathrm{FM}\left(a E_{0} \sin 2 \boldsymbol{k} s\right)$ 。

在应用方面, AM 很容易从被光电二极管探 测到的强度 (或功率) 中得到, 而 FM 由于取决于 光学频率却较难以获得。在互耦合弱区还可发 现, 在两个互相作用的激光器中都有 AM 和 FM, 而且调制项为振幅和频差之比 ${ }^{[4]}$ 。这时耦合系 统可被看作是特殊的相干探测接收器, 也被称作 注人式探测器 ${ }^{[5]}$ 。因为远距离反射镜可看作是 反馈源, 所以基于自耦合的方案就有多种,如后反 馈、诱导调制、注人和自混合等干涉仪。

实现 SMI 的原理较为简单。通常使用的探 测器即为光电二极管, 它在激光二极管制作过程 中被封装在激光器内部。在前输出端仅仅需要一 个准直光学元件, 再用一个衰减器调整返回信号 的强度。通常目标无需是反射型的, 因为从普通 散射面返回的信号已足够大了。

在腔内自混合过程中, 出射功率可表示 为 $^{[6]}$ :

$$
P=P_{0}\left[1+m_{\mathrm{A}} \cdot F(\phi)\right],
$$

式中: $P_{0}$ 是未受干扰时的功率, $\phi=2 \mathrm{ks}$ 是到目标 并返回的距离 $s$ 对应的光学相位, $\boldsymbol{k}=2 \pi / \lambda$ 是波 矢, $m_{\mathrm{A}}$ 是振幅调制因子, 其表达式 ${ }^{[6-7]}$ 为: $m_{\mathrm{A}}=$ $A^{-1 / 2}[c / 2 s(\gamma-1 / \tau], \gamma$ 是增益系数, $\tau$ 是腔的衰 减时间。注意 $A^{-1 / 2}$ 的关系清楚地表明 SMI 过程 取决于场而不是功率, 所以它是一个相干过 程 ${ }^{[5]}$ 。式(1) 中的 $F$ 取决于耦合强度, 是以 $2 \boldsymbol{k s}$ 为变量、以 $2 \pi$ 为周期的函数。所以功率波形的 变化可反映目标距离的变化量 $2 k \Delta s=2 \pi$, 或 $\Delta s$ $=\lambda / 2$, 即与通常的干涉仪相同的半波长。在非 常弱的耦合强度下, $F$ 是 $\phi$ 的余弦函数; 在弱或中 耦合强度下, $F$ 的余弦波形发生了变形,如图 2 所 示。为描述耦合强度, 引人一个注人参数 $C^{[8]}$ 为:

$$
C=\left(1+\alpha^{2}\right)^{1 / 2} A^{-1 / 2} \boldsymbol{k s} / n_{\text {las }} I_{\text {las }},
$$

式中: $k=\varepsilon \delta\left(1-R_{2}\right) / \sqrt{R_{2}}$ 是场耦合返回且与激 光模互相作用的部分, $\alpha$ 是激光器的线宽增强因 子, $\varepsilon$ 是模叠加因子, $R_{2}$ 是输出反射镜的反射率, $\delta$ 是 (场) 目标散射率, $s$ 是目标距离, $n_{\text {las }}$ 和 $I_{\text {las }}$ 分别 是等效折射率和激光腔长。

关于 SMI 信号的波形 $F(\phi)$, 在弱耦合 $C<$ 0.01 情况下, $F$ 开始是在常用干涉仪中出现的余

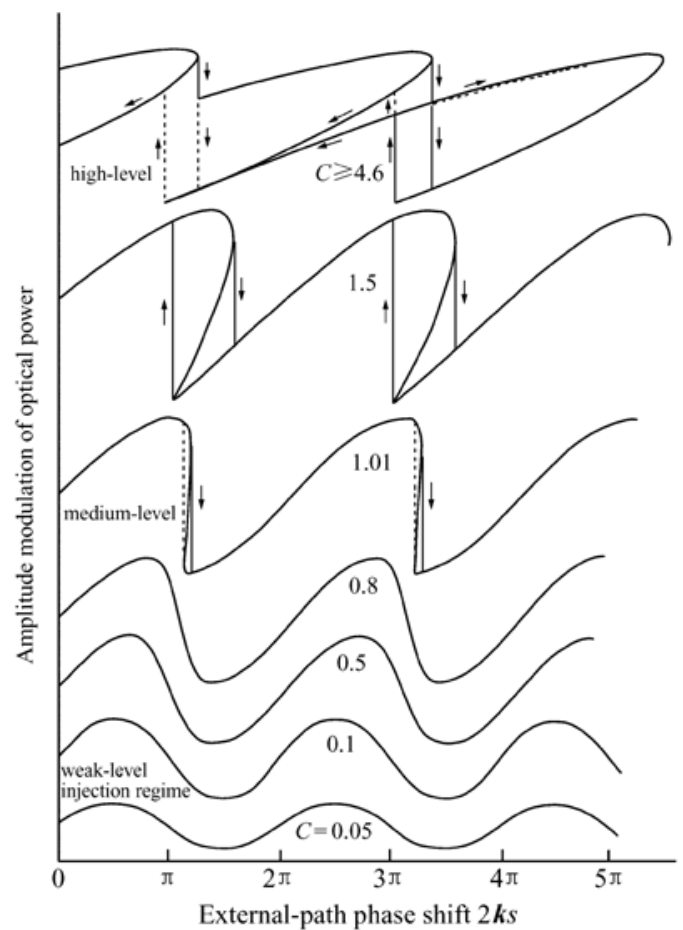

图 2 SMI 从弱到中耦合的波形: 至 $C \approx 0.05$ 时, 波形类 似于光学相移的余弦函数; 增加 $C$, 波形逐渐变 形, 直到 $C=1$, 此时在尾缘出现突变现象, 标志着 $\operatorname{ECM}$ (外腔模式) 开始。上述机制通常可应用于 双通道干涉仪, $\operatorname{LDV}(C \ll 1)$, 条纹计数 $\operatorname{SMI}(1<C$ $<4.6)$, 以及 $\alpha$ 因子、线宽测量及角度测量。在 $C$ 值较大时, SMI 波形首次展现出每个 $2 \boldsymbol{k} s$ 周期超 过一个突变点, 接着突变很快就变成了古怪的形 式, 从而进人了振荡的混沌机制, 这对 SMI 来说 是不利的,但是却可用于密码术

Fig. 2 SMI waveforms for weak to moderate level of coupling: up to $C \approx 0.05$ the waveform is much a cosine function of the optical phase shift, then at increasing $C$ it becomes progressively distorted until at $C=1$ a switching appears in the trailing edge, marking the onset of $\operatorname{ECM}$ ( external cavity mode). Regimes shown are those used in: twochannel interferometer, $\operatorname{LDV}(C \ll 1)$, fringecounting $\operatorname{SMI}(1<C<4.6)$, and also alfa-factor, line width measurement, and angle measurements. At still larger values of $C$, the SMI waveform first displays more than one switching per period of $2 \boldsymbol{k s}$, and then multiple switching soon becoming erratic, entering the chaos regime of oscillations, no more convenient for SMI but useful for cryptography 
弦波,但是随着 $C$ 的增加, 波形逐渐被破坏, 后缘 比前缘陡(见图 2) ${ }^{[3]}$, 直到临界条件 $C=1$, 此时 在尾缘出现了突变现象。对应于中等耦合强度 时,SMI 每个周期出现一个突变, 这是 SMI 工作时 的首选条件。继续增加 $C$, 当 $C=4.6$ 时, 在一个 $2 k s$ 周期内出现两个突变。进一步增加耦合会增 加突变数, 直到波形变得很古怪, 因为在多突变点 中选择哪一个严格地取决于系统的初始条件一即 系统进人多稳态和混沌机制。应用于干涉测量 时, 应使 $C<4.6$, 但是通常在 $C \ll 1$ 的情况下, 信 号处理比较容易。

信号采集可采用图 1 所示方式, 即在激光器 的后输出端进行, 制造商往往在那里提供一个具 有倾斜表面的光电二极管 (PD) 用于监测输出功 率。即便没有后面的 PD, 也可以在输出光束前面 放置一个 PD, 甚至可在目标位置探测 SMI 信号。 如果用 PD 不可行, 尽管这时的 $\mathrm{S} / \mathrm{N}$ 没有后输出 或前输出时的好, 但是还可读取叠加在静止偏置 电压上的 (小) 电压 ${ }^{[9-10]}$ 作为 SMI 信号。

分析自混合现象的第一种方法为利用简单的 旋转矢量叠加模型 (见图 1), 将 AM 和 FM 解释 为正弦和余弦形式的 SMI 信号。第二种方法为 三反射镜模型, 它是将目标看成是激光器的第三 个反射镜, 应用振荡的稳态巴克豪森条件, 得到等 式: $v=v_{0}+\left(c / 4 \pi L n_{1}\right) a \cdot \sin 4 \pi(v / c) s$, 当 $C<1$ 时, 只有一个解, $1<C<4.6$ 时有 3 个解, 其中一 个解不稳定 (中心), 另外两个解稳定 ${ }^{[6]}$, 对应于 波形的一个突变以及 $\operatorname{ECM}$ (外腔模式) 的开始, 在 $C=1$ 时被激活, 并且整个模数增加约为 $2 C / \pi$ 。 上述等式可变为标准的 Adler 频率等式 ${ }^{[4,6,11]}$, 即:

$$
\omega \tau=\omega_{0} \tau-C \sin (\omega \tau+a \tan \alpha),
$$

式中: $\tau=2 L / c$ 是外部时间延迟, $C$ 是耦合因子, $\alpha$ 是线宽增强因子。式(3) 也可从 Lang-Kobayashi 等式中得到。三反射镜模型解释了一些有趣的现 象, 但是缺乏对现象物理条件的详细解释, 例如材 料(半导体) 和激光二极管的相互影响。第三种 方法最为复杂, 它基于电场振幅和相位慢变近似 的 Lamb 方程,并用状态集合的叠加等式(如 Lang 和 Kobayashi (L-K) 等式) 加以修正 ${ }^{[12]}$, 最后可得 到 ${ }^{[3,6]}$ :

$$
\begin{gathered}
\mathrm{d} E / \mathrm{d} t=\left[G_{N}\left(N-N_{0}\right)-1 / \tau_{\mathrm{p}}\right]^{1 / 2} E+ \\
(k / \tau) E(t-\tau) \cdot \cos \left[\omega_{0} t+\phi(t-\tau)\right], \\
\mathrm{d} \phi / \mathrm{d} t=\left\{\alpha\left[G_{N}\left(n-N_{\mathrm{thr}}\right)-1 / \tau_{\mathrm{p}}\right]\right\}^{1 / 2}+ \\
(k / \tau) E(t-\tau) / E(t) \sin \left[\omega_{0} t+\right. \\
\phi(t)-\phi(t-\tau)],
\end{gathered}
$$

$(\mathrm{d} / \mathrm{d} t) N=J \eta / e d-N / \tau_{\mathrm{r}}-G_{N}\left(N-N_{0}\right) E_{\mathrm{i}}^{2}(t)$, 式中 (给出一般值) : $\tau=2 s / C=30 \mathrm{~ns} / \mathrm{m}$ 是往返光 程延迟; $G_{N}=8.1 \times 10^{-13} \mathrm{~m}^{3} / \mathrm{s}$ 是模式增益; $k$ 是场 耦合与振荡模之比; $N$ 为载粒子密度 $\left(\mathrm{m}^{-3}\right)$; $N_{\mathrm{thr}}=2.5 \times 10^{24} \mathrm{~m}^{-3}$ 是所在阈值时的载粒子密度; $N_{0}=1.2 \times 10^{24} \mathrm{~m}^{-3}$ 是反转时的载粒子密度; $\tau_{\mathrm{p}}=$ $2 \mathrm{ps}$ 是腔内光子寿命, $\tau_{\mathrm{r}}=5 \mathrm{~ns}$ 是载粒子寿命, $\alpha=$ $3 \sim 6$ 是线宽增强因子; $\omega_{0}=k / c$ 是未受扰动的频 率; $J$ 是百浦电流密度和在厚度为 $d$ 的激活层内 部的量子效率。L-K 等式是各点独立的等式, 描 述了受激材料,它与基于三反射镜的系统等式有 很大的不同, 通过被 $k$ 相乘的耦合项以及延迟项 $E(t-\tau)$ 和 $\phi(t-\tau)$ 完成了对激光振荡的描述。 利用 L-K 方程可得到其它方法所能得到的所有 结论,包括 Adler 的等式 (见式 (3)), 而且还揭示 了 $\mathrm{AM} / \mathrm{FM}$ 调制, $F(\phi)$ 的波形, 突变时的 $C$ 因 子 $^{[3]}$, 初始的双稳态和多稳态, 谱线变宽或变窄, 规律到混沌 ${ }^{[13]}$ 等现象。与实验比较, L-K 等式给 出了较为精确的弱水平下的 SMI 现象和高水平 时与混沌有关的动力学模型。唯一的偏离是比预 期的振荡线宽要宽, 为了解释这一现象, 在式 (4) 的第二行引人了一个线宽增强因子 $\alpha^{[14]}$ 。

\section{SMI 千涉仪的特点}

与其他传统干涉仪比较, SMI 具有如下优势:

(1) 光学零件数量少 (在激光器外部不需其 它光学元件);

(2) 光路自排列 (SMI 可在激光点到达之处 完成测量);

(3) 不需要空间、波长或杂散光的滤波器 (激 光腔作为滤波器);

(4)工作在普通的散射目标面 (SMI 允许强 损失);

(5) 信号可在光束的任何位置, 包括目标的 边缘 (这是 SMI 独有的特征); 
(6) 分辨率为条纹计数的 $\lambda / 2$, 用模拟处理方 法可达到亚波长量级 (可达到探测的量子噪音 限) ;

（7）带宽可达几百千赫兹或兆赫兹。

当然,SMI 也存在一些不足:

(1) 在基本光路中没有参考臂;

(2) 由于采用 F-P 激光二极管, 波长精度和 稳定性差;

(3) 结构灵活性差;

(4) 工作在散射目标表面,SMI 信号受到散斑 图统计特性的影响, 会引起振幅衰减以及相位误 差。

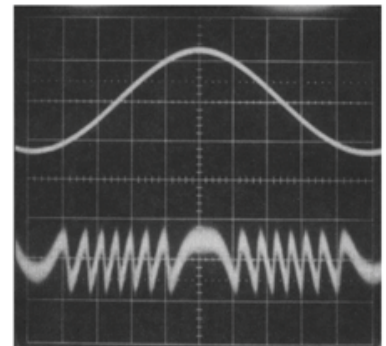

(a) $C=0.6$

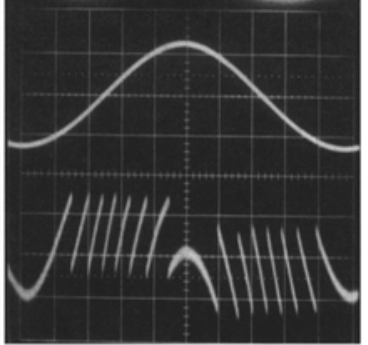

(b) $C=2.2$
图 3 在目标信号 $s(t)$ 为正弦驱动信号时 (上曲线) 得到的 SMI 实验信号 (下曲线)。左: 在 $C=$ 0.6 时 $\cos 2 \boldsymbol{k} s$ 波形有点变坏,下降的半周期比 上升的快; 右: 在 $C=2.2$ 时, 在下降的半周期 发生突变。波长为 $850 \mathrm{~nm}$, 时间尺度为 $2 \mathrm{~ms} / \mathrm{div}$ 。光电二极管探测的波形中目标每移 动 $\lambda / 2$ 会有个突变。目标后退时 (上曲线) 突 变是负走向(下曲线), 目标前进时是正走向。 (见文献 [6])

Fig. 3 Experimental signals of the SMI (lower traces), obtained in response to sinusoidal drive $s(t)$ of the target (upper traces). Left: at $C=0.6$, the $2 \boldsymbol{k} s$ waveform is slightly distorted, with falling semi-periods somewhat faster than the rising ones; right: at $C=2.2$, a switching occurs in the fall semi-period. Wavelength is $850 \mathrm{~nm}$, time scale: $2 \mathrm{~ms} /$ div. Note that the waveform detected by the photodiode has switching every $\lambda / 2$ displacement of the target. The switching is negative going (lower trace) when the target is receding ( upper trace) and is positive-going when the target is approaching. (Ref. [6])
一般地, 从 SMI 的示意图 (见图 1) 和波形 (见图 3), 可能会想到一个简单的处理方案就可 以适合所有的应用,但事实并非如此。首先,通过 数波的峰-峰 $I_{\mathrm{p}-\mathrm{p}}$ 来计算周期数, 它对应目标距离 $\Delta s$ 的 $\lambda / 2$ 变化,从而实现 $I_{\mathrm{ph}}(t)$ 的数字处理。其 次可以对 $I_{\mathrm{ph}}(t)$ 进行模拟处理, 因为观察得到小 的 $\Delta I_{\mathrm{ph}}$ 变化对应于小的距离 $\Delta s$ 变化, 这很容易从 $\Delta s=(\lambda / 2) \Delta I_{\mathrm{ph}} / I_{\mathrm{P}-\mathrm{P}}$ 等式中发现。最小的 $\Delta I_{\mathrm{ph}}$ 比 $I_{\mathrm{P}-\mathrm{P}}$ 小得多, 从图 3 中的迹线可知, 平均噪音 $\sigma_{\mathrm{IPh}}$ 与 $I_{\mathrm{ph}}$ 相关, 用模拟处理方法可使分辨率变得更 好, 很容易达到纳米量级, 在实验中达到了 $20 \sim$ $50 \mathrm{pm}$ (量子噪音限甚至更小, 可达到 $\left.10 \mathrm{fm} / \sqrt{\mathrm{Hz}}^{[5]}\right)$ 。到目前为止, 动态范围较大时, 数字处理结果比较满意。数 $\lambda / 2$ 条纹显示, 测量 的动态范围仅受分配到计数器中的十进制数的限 制, 所以很容易达到 $\lambda / 2$ 的 $10^{5} \sim 10^{6}$ 倍的步长, 相 应地对于 $I_{\mathrm{P}-\mathrm{P}}$ 波形,对应的模拟量是 $\lambda / 2$ 。当采用 数字处理方法, 在测量范围较大 (一般为几米) 并 要求达到亚波长分辨率情况时, 习惯上将 SMI 归 为位移测量仪器 ${ }^{[15]}$ 。另一方面, 用模拟方法处理 的 SMI, 可能对分析小振幅的 (如 1 100 的峰峰) 周期运动更有利, 在分析机械振动或机械疲 劳时 ${ }^{[5]}$, 可将它归为振动测量仪器。有些作者把 测振仪当作测速计, 这显然不是一个很好的选择, 因著名的激光多普勒或 LDV 仪器 ${ }^{[3]}$ 用于流场分 析和风力测定 ${ }^{[3]}$ 更有优越性。当然, SMI 也可被 设计成实现测速计功能的仪器。

\section{SMI 千涉仪的应用}

在最近的 20 年里,报道有关 SMI 的应用主要 包括：

(1) 位移、振动、速度、角度测量;

(2) 线宽、 $\alpha$ 因子等激光参数的测量;

(3) 厚度、折射率、粗粘度、机械共振、力/应 力迟滞等物理量的测量;

(4) 远距离回波探测、返回损耗、共焦显微镜 传感器、生物运动性等传感测量。

\section{1 位移测量}

在机械量计量中应用的仪器是基于稳频 $\mathrm{He}$ $\mathrm{Ne}$ 激光器的“激光干涉仪” [5]。当使用角雉棱镜 
作为反光器件时, 在几米的位移范围内, 仪器分辨

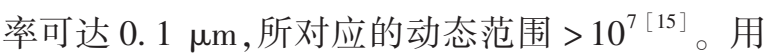
于位移测量的 SMI 能以散射模式工作、可测量未 经处理的表面、可替代角雉棱镜、且费用低廉、容 易操作。普通的激光干涉仪需要两路信号, 即 $\cos 2 \boldsymbol{k} s(t)$ 和 $\sin 2 \boldsymbol{k} s(t)$, 以便能够确切地获得正 弦/余弦函数的相位并正确地提取 $s(t)$ 。与之相 比,SMI 最大的优势是在 $C>1$ 区域很容易得到位 移增量信号。典型的 SMI 结构信号波形如图 3 所 示, 可以看出外部距离 $s(t)$ 每变化 $\lambda / 2$, 波形信号

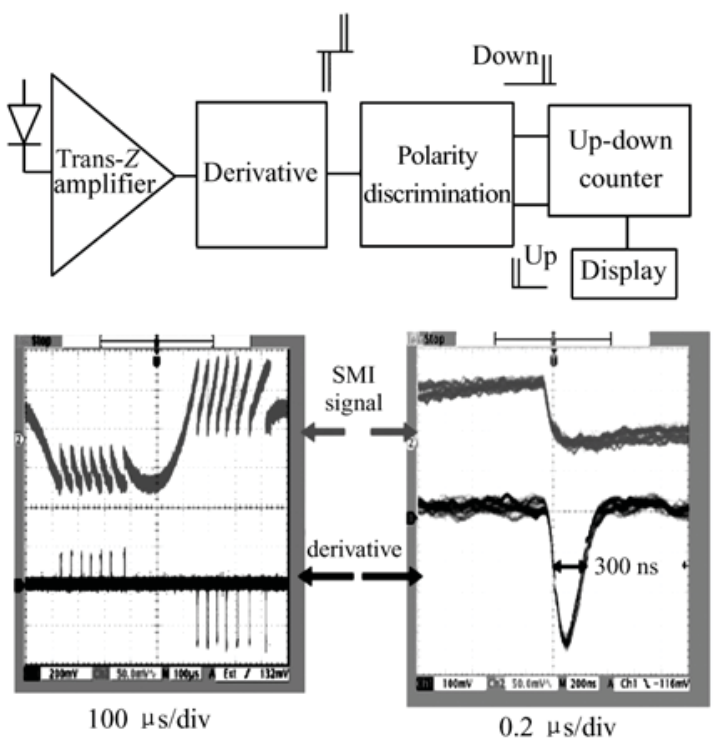

图 4 分辨率为 $\lambda / 2$ 的位移测量 SMI 示意图, 其对应 波形的 $C>1$ : 光电二极管的信号通过跨阻放 大器、时间微分、脉冲整形存储到十进制计数 器的上/下输人中, 用一个乘法器输出十进制 米单位的最终结果。典型的分辨率是 0.5 $\mu \mathrm{m}$, 目标最大速度在脉宽 $300 \mathrm{~ns}$ 时为 $1.2 \mathrm{~m} / \mathrm{s}$

Fig. 4 Schematic of an SMI for measuring displacement with $\lambda / 2$-resolution from the waveforms with $C>1$ : the photodiode signal is passed through a trans-impedance op-amp, time-differentiated and pulses rectified and sorted to the $\mathrm{Up} /$ Down input of a decimal counter. A multiplier (not shown) is used to bring the accumulated counting to metric decimal on the display. Typical resolution is $0.5 \mu \mathrm{m}$, and maximum speed of the target, with a pulse width of $300 \mathrm{~ns}$, is about $1.2 \mathrm{~m} / \mathrm{s}$
突变一次。目标向着光源前进 (后退) 时发生正 (负) 走向的突变表示计数时位移信号 $s$ (加或减) 的符号 ${ }^{[6,16]}$ 。图 4 为用于位移测量的 SMI 结构。

光电二极管的输出经过跨阻放大器放大后, 将 $I_{\mathrm{Ph}}$ 转换成电压, 然后对时间进行微分, 以提取 脉冲的突变点, 判断脉冲的 $(+/-)$, 将它们送到 分开的输出端。采用十进制加减脉冲 ${ }^{[6]}$, 计数内 容是从计数器复位时刻 $t=0$ 开始到当前时间为 止目标的累计位移 $s(t)$, 单位是 $\lambda / 2$ 。为了以十 进制、 $m$ 单位读出, 需有一个以 $\lambda / 2$ 为计数单位 的乘法器。在脉宽为 $300 \mathrm{~ns}$ 时, 目标最大速度为 $1.2 \mathrm{~m} / \mathrm{s}$ 。

采用 $850 \mathrm{~nm} \mathrm{GaAlAs}$ 激光二极管, 其分辨率 约为 $0.5 \mu \mathrm{m}$, 可满足大部分机床定位和测量要 求 ${ }^{[15]}$ 。而且, 如果维持微分器处理时间常数足够 短 $(\tau=300 \mathrm{~ns}$ ), 就可得到每秒约为 $1 / \tau$ 脉冲 (即 $\left.3 \times 10^{6}\right)$, 计数脉冲最大目标速度是 $v=\lambda / 2 \tau \approx$ $1.2 \mathrm{~m} / \mathrm{s}$ 。即使对一个散射不好的目标, SMI 信 号也能被探测到, 且能够处理的距离可达到 $2 \mathrm{~m}^{[6,16]}$ 。

在考虑测量的准确度和精密度时, 波长稳定 性是首要考虑的问题。在实验室环境下,仔细控 制偏置电流和温度可使工作时的稳定性达到 $10^{-6}$ 。散斑图的统计特性会影响 SMI 信号的振幅 并引人相位误差 ${ }^{[3]}$ 。为了评估 SMI 的固有特性, 这里采用角雉立方棱镜作为目标物进行了一组位 移 $(s=65 \mathrm{~cm})$ 的重复性测量。

如图 5 所示, 相对误差 $\delta s / s$ 为 $-95 \times$ $10^{-6} /{ }^{\circ} \mathrm{C}$ 。采用热电冷却器稳定激光器芯片温度 后, 数据回到零线附件, 在 $4 \mathrm{~h}$ 内采样 60 组数据, 误差范围约为 $2 \times 10^{-6[16]}$ 。

实际上, 在应用 SMI 测量位移时, F-P 激光器 分辨率达不到 $10^{-6}$ 水平, 因为在开启后激光器变 热从而出现波长的模式跳跃 (每个能达到 $\Delta \lambda=$ $1 \mathrm{~nm})$, 同时对 $\lambda$ 产生迟滞现象 ${ }^{[3]}$ 。这时可采用 分布反馈 $($ DFB ) 激光器替代 F-P 激光器, 这样光 源在实验室条件下可长期保持 (大于 1 年) $10^{-6}$ 的精确度 ${ }^{[16-17]}$ 。在 SMI 结构中可采用一般的散 射体代替角雉棱镜, 而对基于 $\mathrm{He}-\mathrm{Ne}$ 激光器的干 涉仪来说是不允许的 ${ }^{[3]}$ 。 


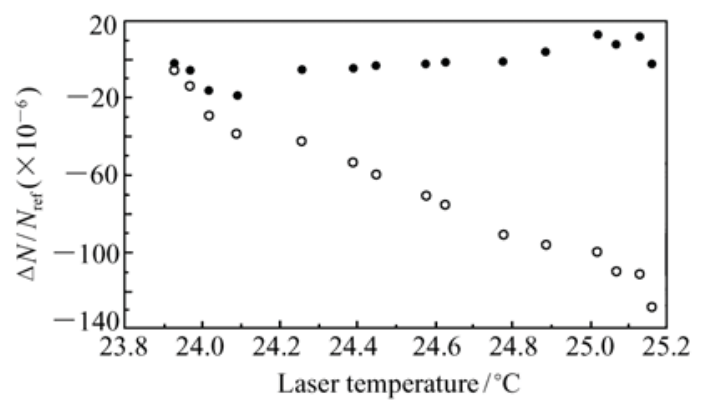

(a)

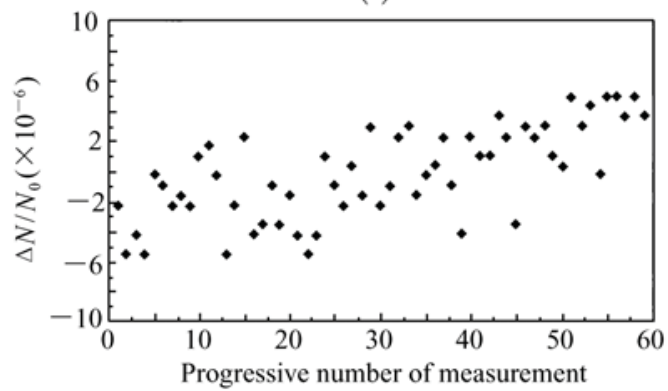

(b)

图 5 测量 $65 \mathrm{~cm}$ 位移时给出的激光二极管温度函 数 ( (a)中的空心圆)。温度稳定后, 数据在零 点附近 ( ( a ) 中的实心圆)。在 $4 \mathrm{~h}$ 内测量了 60 个点, 误差约为 $\pm 2 \times 10^{-6}(\mathrm{~b})$ ( 见文献 [16])

Fig. 5 Results of measuring a $65 \mathrm{~cm}$ displacement exhibit a roll-off as a function of diode laser temperature (open circles of (a)). After temperature is stabilized, data return around zero (full dots of (a)). The spread over a $N=60$ sample of measurements lasting 4 hours is about $\pm 2 \times$ $10^{-6}$ (b) (see Ref. [16])

通常散斑图影响返回激光腔场的振幅和相 位 ${ }^{[18]}$, 分析结果显示 ${ }^{[3]}$, 相位误差相对较小, 在 $s=1 \mathrm{~m}$ 时仅仅有几个波长的起伏,但振幅变化较 严重, 由此会引起信号丢失, 从而丢失与 $\lambda / 2$ 有 关的计数,应该加以抑制。这种状况通常在测量 目标沿被测路径的位移 $s(t)$ 对应的散斑比较 “暗”的情况下发生。利用统计特性可减轻散斑 变暗: 暗散斑周围可能有其它较亮的散斑。如果 让投射到目标上的光点发生一个小的偏离, 偏离 量大到足够改变散斑采样,但小到测量距离不变, 就可能避免“暗”散斑现象。为了追踪亮散斑,可 用装有物镜的两个 PZT 压电激励器驱动, 使之沿 $X-Y$ 轴运动,用伺服电路形成闭环, 将探测信号反
馈给压电器以确保 SMI 信号达到最大 ${ }^{[19]}$ 。该技 术被称为亮散斑追踪 (BST), 图 6 给出了一个使 用该技术的例子。
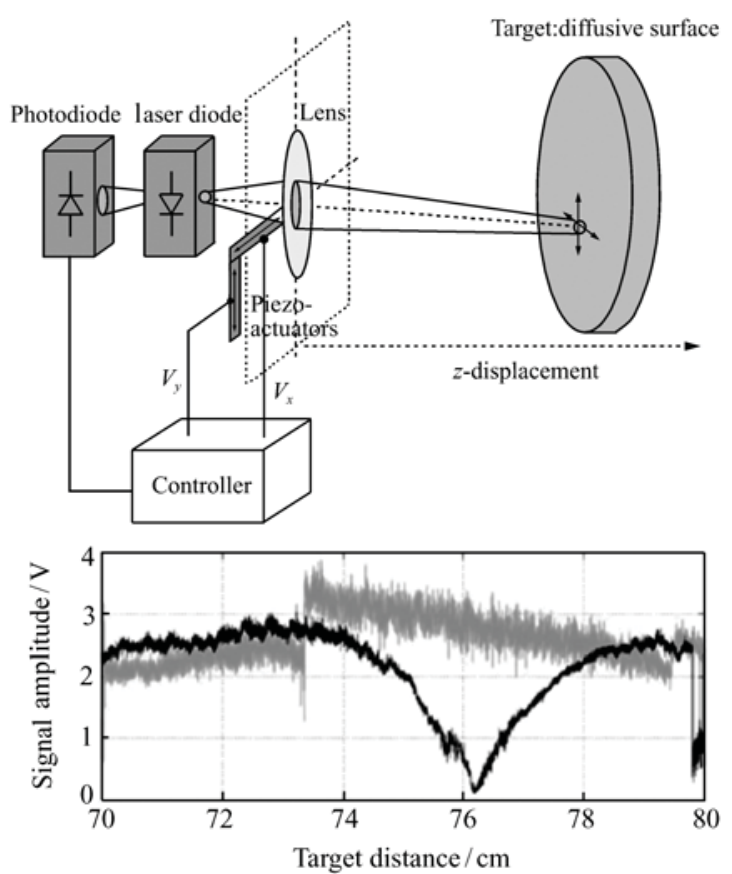

(a)

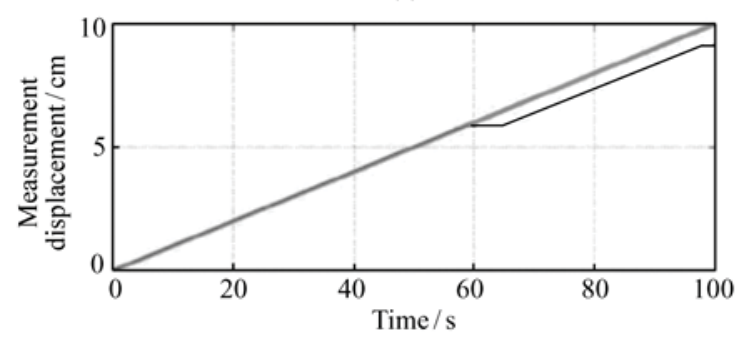

(b)

图 6 (a) BST 技术一亮散斑追踪: 物镜在一对 PZT 驱动控制下可沿 $X$ 和 $Y$ 方向移动以跟踪区域 强度最大值。(b) 证明 BST 控制的实验结果, 避免了暗散斑在 $s=76 \mathrm{~cm}$ 处的计数丢失 (见 文献 $[19])$

Fig. 6 In the technique called Bright Speckle Tracking (BST), the objective lens is moved slightly along the $X$ and $Y$ axes by a pair of PZT actuators so as to track the local maximum of intensity scattered by the diffuser back into the laser(a). In an experiment demonstrating BST control, a dark speckle affecting a counting loss at $s=76$ $\mathrm{cm}$ is avoided and the corresponding error is removed(b). (see Ref. [19]) 
在一般工作条件下, “暗” 散斑通常出现在 $s$ 为 $74 \sim 78 \mathrm{~cm}$ 之间, 这时的振幅较小, 计数易丢 失。打开 BST 电路, 避免 $76 \mathrm{~cm}$ 处的下降点, 正确 计数。这证明了 BST 控制避免了暗散斑在 $s=$ $76 \mathrm{~cm}$ 处的计数丢失。在图 6 中还可以看到, $s=$ 73.5 处有一个陡峭的上升, 在此处系统跳到了附 近亮的散斑处。严格地讲,用 BST 可以减少但不 能够完全消除散斑变暗的可能性。然而, 如果让 $\boldsymbol{k}$ 从 0.01 下降到约为 $10^{-6[19]}$, 则 SMI-BST 仪器 可应用于非散射体, 可作为变化范围为 $\mathrm{m}$ 量级、 分辨率为 $\lambda / 2$ 的便携式仪器 ${ }^{[20]}$ 。

\section{2 振动测量}

如果被测量的位移量是小振幅的周期运动且 频率范围从声波到 $\mathrm{MHz}$, 则以 $\lambda / 2$ 步长计数过于 粗鉌, 这时一般采用模拟方法处理信号 $s(t)$ 。起 始时模拟形式受动态范围限制, 因为运算放大电 路可支持的信号范围从毫伏 (偏置限) 到数十伏, 或有 $10^{4}$ 的动态范围, 所以比具有 $10^{6} \sim 10^{7}$ 动态 范围的数字处理位移干涉仪要少 $10^{2} \sim 10^{3}$ 量级。 但是用模拟信号处理方法,对小位移可以大幅提 升灵敏度, 不受半波长限制, 仅受到探测信号噪音 的限制, 该限制也被称作噪音等效位移 (NED), 可容易地从探测信号 $I_{\mathrm{ph} 0}(1+\cos \phi)$ 中获得 ${ }^{[5]}$, 其 中 $\phi=2 \boldsymbol{k} s$, 在半条纹点 $(\phi=\pi / 2)$ 处具有最大相 位灵敏度, 即为 $\left(\Delta I_{\mathrm{ph}} / I_{\mathrm{ph} 0}\right)^{2}=(\Delta \phi)^{2}$ 。回顾一下 与探测电流有关的肖特噪音表达式, 即 $\left(\Delta I_{\mathrm{ph}}\right)^{2}=$ $2 \mathrm{e} l_{\mathrm{ph} 0} B^{[5]}$, 式中 $B$ 是测量的带宽, 很容易得到: $\left\langle(\Delta \phi)^{2}\right\rangle=2 \mathrm{e} B / I_{\mathrm{ph} 0}=\mathrm{SNR}^{-1}$, 这里 SNR 是幅值 (如光电流) 测量的信噪比。利用 $\phi 2 k \Delta s$ 和 $\boldsymbol{k}=$ $2 \pi / \lambda$ 可得到 ${ }^{[5]}$ :

$$
\begin{gathered}
\mathrm{NED}=\left\langle\Delta s^{2}\right\rangle^{1 / 2}=\lambda / 2 \pi\left[\mathrm{e} B I_{\mathrm{ph} 0}\right]^{1 / 2}= \\
\lambda / 4 \pi \mathrm{SNR}^{1 / 2} .
\end{gathered}
$$

由式 (5) 可知 ${ }^{[5]}$ : 对应 $\mu \mathrm{A}$ 的探测电流和 $\mathrm{MHz}$ 的带宽可探测最小 NED 达到 $\mathrm{nm}$ 量级, 对 $\mathrm{mA}$ 和 $\mathrm{kHz}$ 甚至可达 $\mathrm{pm}$ 量级,这实际上已经达到 或接近极限水平, 为解决在处理电路中普遍存在 的大量大扰动和干涉源提供了手段。还有一些因 素限制了干涉仪可测的最小信号, 如相干、散斑和 热动态变化, 但是经过优化设计后 SMI 可以忽略 这些因素 ${ }^{[5]}$ 。

目前通过模拟信号实现小信号的振动测量主
要有两种方法: (1) 在半条纹处读出, 以便利用干 涉仪读出相位-电流关系的线性变换。为了达到 这一目的, 设置干涉仪的静态工作点在余弦幅值 之间, 即 $\phi=\pi / 2$ 附近, 使 $\phi=\pi / 2+2 k s$, 对于小 位移则有: $\Delta I_{\mathrm{ph}}=-I_{\mathrm{ph} 0} \Delta \phi=-I_{\mathrm{ph} 0} 2 \boldsymbol{k} \Delta s$, 例如 $\Delta s$ 和 SMI 输出信号 $\Delta I_{\mathrm{ph}}$ 的线性关系可直接从探测到

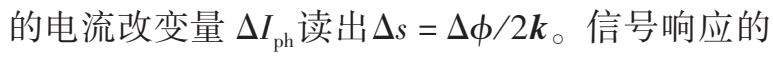
线性范围被余弦状函数限制在 $\lambda / 2$, 对于早期的 传统干涉仪 ${ }^{[5]}$ 由于有参考臂很容易实现该技术, 这时半条纹条件可表达为 $\cos \left(\phi_{\text {mess }}-\phi_{\text {ref }}\right) \approx 0$ 。 为了实现这一条件, 调节参考光程使得 $\phi_{\mathrm{ref}}=$ $\Delta \phi_{\text {mess }}+\pi / 2$, 从而对 $\Delta \phi_{\text {mess }}$ 有 $\cos \left(\phi_{\text {mess }}-\phi_{\text {ref }}\right)=$ $-\sin \Delta \phi_{\text {mess }} \approx-\Delta \phi_{\text {mess }}$ 。（2)波前重构技术。通过 $0<2 \boldsymbol{k} s<2 \pi$ 时的转换关系 $I_{\mathrm{ph}}=I_{\mathrm{ph} 0}[1+$ $F(2 k s))]$, 并利用展开算法对 $N 2 \pi<2 \boldsymbol{k} s<(N+$ 1) $2 \pi$ 时的情况进行扩展重构, 可以从测量数据

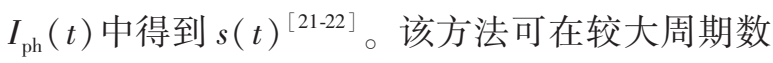
$N$ 时重构 $s(t)$, 仅受限于 SMI 的参数 $C$ 和 $\alpha$ 准确 度, 见式(3) 和式 (4)。实际上, 文献中给出的 $N$ 值在 30 100 之间, 或对应的最大振幅 $s$ 在 50 $150 \mu \mathrm{m}$ (峰-峰)之间, 同时对小 $s$ 残余计算误差为 $5 \sim 10 \mathrm{~nm}^{[21]}$, 远远大于方法 (1) 中达到的噪音限。

\subsection{1 小振幅振动}

在 SMI 中没有参考通道可以调节条纹信号 的象限, 但是可以利用半导体激光器波长依赖于 偏置电流这一关系开发一个控制环, 并将其工作 点设在干涉仪的半条纹处 ${ }^{[23]}$ 。

在中反馈区 $(C>1)$ 可以利用条纹的慢半周 期, 在这里工作区域近似为线性 (见图 $7(\mathrm{a})$ )。 为了动态设置半条纹的工作点, 须考虑光电二极 管互阻放大器输出端的探测信号以及它的幅度变 化。设 $V_{\text {ref }}$ 为半条纹电压, 为了锁住信号起伏的中 间静态点, 将 $V_{\text {ref }}$ 作为差分运算放大器 (图 7 中的 增益 $\mathrm{A}$ 模块) 的参考输人, 在另一输人端接收探

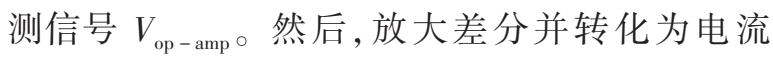
(图 7 中的 $G_{\mathrm{m}}$ 模块), 并反馈到激光二极管。电 流 $I_{\text {bias }}$ 引起波长变化 $\Delta \lambda=\alpha_{\lambda} \Delta I_{\text {bias }}$, 从而导致波数 变化 $\Delta \boldsymbol{k}=-\boldsymbol{k} \Delta \lambda / \lambda$, 关闭反馈环并伺服相位 $2 \boldsymbol{k s}$ 信号。目标移动产生相位 $2 \boldsymbol{k} \Delta s$, 反馈环和波长变 化的相互作用得到一等值并反向的相位 $-2 s \Delta \boldsymbol{k}$ 。 经历反馈环后, 振动信号 $2 k \Delta s$ 在差分放大器的输 


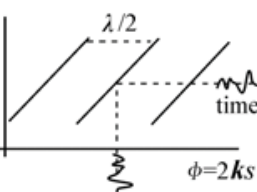

(a)

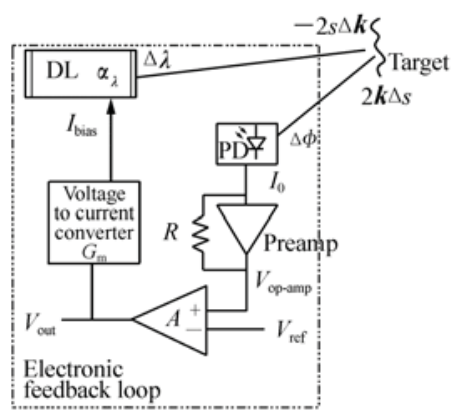

(b)
图 7 小振幅振动仪中的线性处理: 为了锁住信号起 伏的中间静态点, 用半波电压 $V_{\text {red }}$ 作为差分放 大器预放大信号 $V_{\text {op - amp }}$ 的参考信号 (a)。将输 出转换为电流并提供给激光二极管。由于目 标移动造成了相位变化 $2 \boldsymbol{k} \Delta s$, 电子反馈环作 用使得波长发生变化, 从而给出一等量的反相 位 $-2 s \Delta \boldsymbol{k}$ 。在差分放大器 $\mathrm{A}$ 的输出端得到振 动信号 $2 \boldsymbol{k} \Delta s(\mathrm{~b})$ (见文献 [23])

Fig. 7 Linear processing in a small-amplitude vibrometer: to lock the quiescent point at the middle of the signal swing (a), the half-fringe voltage level $V_{\text {ref }}$ is used as the reference of the difference amplifier receiving the preamp signal $V_{\mathrm{op}-\mathrm{amp}}$. Output is then converted to current and feeds the laser diode. To a phase variation $-2 s \Delta \boldsymbol{k}$ due to target motion, the electronic feedback loop reacts with a wavelength change giving an equal and opposite phase $-2 s \Delta \boldsymbol{k}$. The vibration signal $2 k \Delta s$ is then found at the output $V_{\text {out }}$ of the difference amplifier A(b). (see Ref. [23])

出端输出 $V_{\text {out }}($ 图 7(b)), 这是由大的环增益造成 的。 $V_{\text {ref }}$ 和运算放大器输出 $V_{\mathrm{op}-\mathrm{amp}}$ 之间很小的压 差是产生 $V_{\text {out }}$ 所需的条件,而且它还可使 $\alpha_{\lambda} G_{\mathrm{m}} V_{\text {out }}$ 偏置电流满足零相位条件, 即 $2 \boldsymbol{k} \Delta s=0$ 。所以从 运算放大器输出 $\Delta V_{\text {out }}$ 获得的振动信号为:

$$
\Delta V_{\text {out }}=\left[\alpha_{\lambda} G_{\mathrm{m}}\right]^{-1}(\lambda / s) \Delta s .
$$

该结果不受光电探测器信号 $I_{\mathrm{ph}}$ 的幅值以及 其波动 (包括目标后散射因子和散斑图变暗) 的 影响。唯一的条件是环增益 $G_{\mathrm{loop}}$ 足够大。由 图 7, 环增益 $G_{\text {loop }}=R A \alpha_{\lambda} G_{\mathrm{m}}\left(s / \lambda^{2}\right) \sigma P$, 其中 $\sigma P_{0}=$ $I_{\mathrm{ph} 0}$ 是光电探测平均电流, $\sigma$ 是光电二极管的谱密 度。在一般的测振仪 ${ }^{[23]}$ 结构中, 可让环增益增大
至 $G_{\text {loop }} \approx 500 \sim 1000$,与非反馈条件相比, 在闭环 中残余的非理想情况可被与环增益相等的因子削 弱,特别是散斑图变暗程度被因子 500 1 000 降 低且不再对测量有影响。反馈环的另一个作用是 因子 $G_{\text {loop }}$ 可提高线性和动态范围 ${ }^{[23]}$ 。基于控制 理论, 动态范围极限仅是在环中引人的误差, 所以 小信号振动计不局限于振幅 $\leqslant$ 半条纹 (或 $>\lambda / 2$ ) 的情况。随着信号的增加并趋于超过该条纹, 反 馈环将它拉回来, 留下残余量仅为 $1 / G_{\text {loop }}$ 。动态 范围为 $G_{\text {loop }} \lambda / 2$, 即 $200 \sim 500 \mu \mathrm{m}$ 。

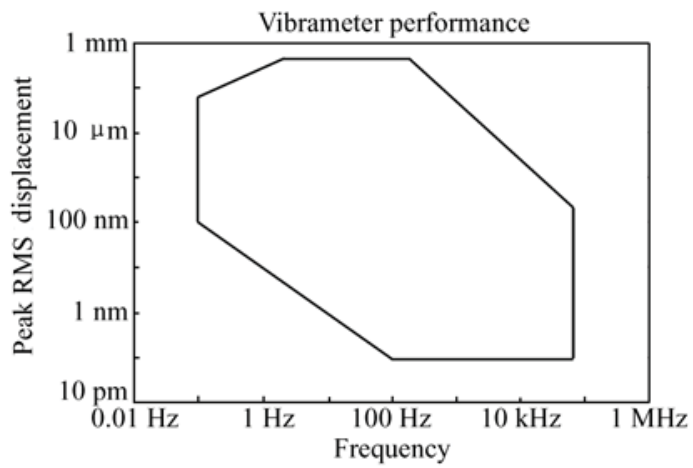

(a)

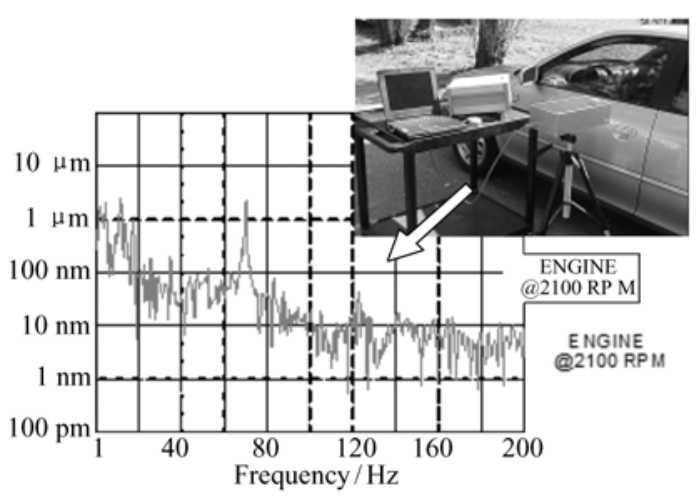

(b)

图 8 (a) 锁定半条纹的振动仪性能: 最小位移信号 是 $100 \mathrm{pm}(B=1 \mathrm{~Hz})$, 最大幅值为 $500 \mu \mathrm{m}$, 频 率为 $0.1 \mathrm{~Hz} \sim 80 \mathrm{kHz}$ (见文献[23]); (b) 现场 探测汽车门上小振动的仪器

Fig. 8 Performance of a half-fringe locked vibrometer (a) : minimum displacement signal is $100 \mathrm{pm}$ $(B=1 \mathrm{~Hz})$ and maximum amplitude $500 \mu \mathrm{m}$, frequency from $0.1 \mathrm{~Hz}$ to $80 \mathrm{kHz}$ (see ref. [23]). The instrument at work detecting small vibrations on the door, as produced by the engine of the car(b) 
按照半条纹伺服环概念研发的宽带振动计性能在 文献 $[24]$ 中进行了报道。该原型机最小可探测 信号 $\mathrm{NED}=100 \mathrm{pm}$ (带宽 $B=1 \mathrm{~Hz}$ ), 最大动态范 围约为 $500 \mu \mathrm{m}$ 。带宽/振幅性能关系见图 8 , 同 时该图还给出了室外场中振动测量的实例。

\subsection{2 大振幅振动}

如果能从被测波形 $I_{\mathrm{ph}}(t)$ 或 $P(t)$ 中通过对 式( 1) 进行转换解出相位 $\boldsymbol{\phi}=2 \boldsymbol{k} s(t)$, 那么从理论 上就可得到不受动态测量范围限制的信号 $s(t)$ 。 完成转换算法分为两部分: 对 $-\pi<\phi<+\pi$, 应去 除式 (1) 中 $F$ 函数中的非线性项, 当 $\phi$ 超过 $2 \pi$ 时, 对相位去包裹确定 $(2 N-1) \pi<\phi<(2 N+$ $1) \pi$ 的范围。当反馈较弱 $(C<1)$ 时,波形是时间 对称的, 为了从式 (1) 中得到明确的解, 需要两个 信号: $\phi=2 \mathrm{ks}$ 的正弦和余弦信号 (参看 4.2.3 节)。大量文献 ${ }^{[25]}$ 论述了应用反余弦函数测量 $I_{\mathrm{ph}}(t)$ 以及其相位去包裹的方法。当反馈较强 $(C$ $>1$ ) 时, 一个信号就足以完成重构, 因为波形中 的突变给出了相位去包裹时增量所需要的符号。 如 S. Merlo ${ }^{[21]}$ 首次提出, 从式 (1) 和式 (3) 可得到 一个算法, 该算法可被用于从实验测量得到的

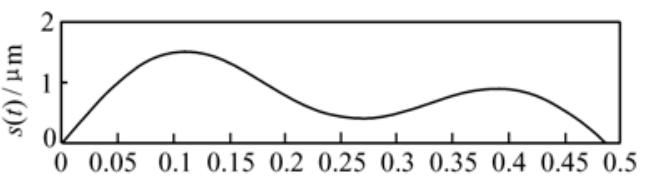

(a) 作用的 $s(t)$

(a) Applied $s(t)$

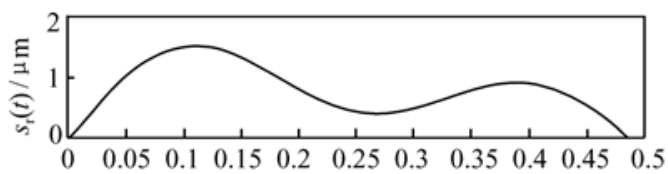

(c) 重构的 $s_{\text {rec }}(t)$

(c) Reconstructed $s_{\text {rec }}(t)$
$F(t)=\Delta P / P_{0}$ (或 $\left.\Delta_{\mathrm{ph}} / I_{\mathrm{ph} 0}\right)$ 的波形中, $s(t)$ 可表 示为:

$$
\begin{gathered}
2 k s(t)=\arccos F(t)- \\
C\left\{-\alpha F(t) \pm \sqrt{\left[1-F(t)^{2}\right]}\right\} / \\
\sqrt{1+\alpha^{2}}+m \pi .
\end{gathered}
$$

对 $\mathrm{d} F(t) / \mathrm{d} t \cdot(\mathrm{d} s / \mathrm{d} t)<0$ 符号应该取 “ $+”$, 对 $\mathrm{d} F(t) / \mathrm{d} t \cdot(\mathrm{d} s / \mathrm{d} t)>0$ 取 “ $-”, F(t)$ 的每两 个零交叉点 $m$ 增加 $1^{[21]}$ 。应用式 $(7)$ 需要确定参 数 $C$ 和 $\alpha$ 。如果线宽增强因子不是太小而且有 $\alpha^{2} \gg 1, C$ 可由 $F(t)$ 波形形状确定 ${ }^{[6]}$, 通过寻找 $F$ 增加 $\left(t_{\text {rise }}\right)$ 和下降 $\left(t_{\text {fall }}\right)$ 的半周期, 可得 ${ }^{[6]}$ :

$$
t_{\text {rise }} / t_{\text {fall }}=\left\{\sqrt{\left(1+\alpha^{2}\right) \pi}-2 C \alpha\right\} /\left\{\sqrt{\left(1+\alpha^{2}\right) \pi}+\right.
$$$$
2 C \alpha\} \cong(\pi-2 C) /(\pi+2 C) \text {. }
$$

另外, $\alpha$ 因子可由激光二极管类型估算也可 按照本文后面所说的方法测量。对一般的法布 里-珀罗多量子阱 (MQW) 激光二极管, $\alpha$ 在 $4 \sim 6$ 间变化, 计算时取 $\alpha=5$, 对非正弦周期波形 $s(t)$ 应用式(7) 和式 (8) 给出的重构结果, 如图 9 所 示。 $2 \mu \mathrm{m}$ 波形的重构误差 $<5 \mathrm{~nm}^{[21]}$ 。

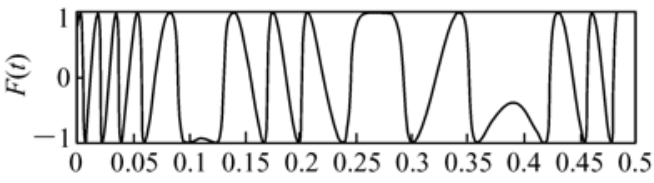

(b) $\mathrm{SMI}$ 波形 $I_{\mathrm{ph}}(t)$

(b) SMI waveform $I_{\mathrm{ph}}(t)$

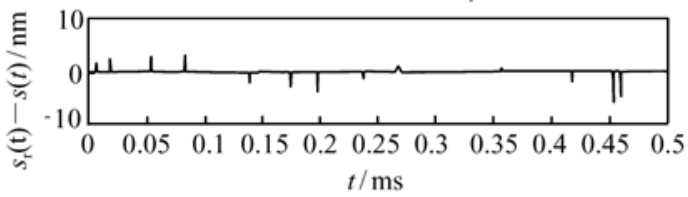

(d) 误差 $s(t)-s_{\mathrm{rec}}(t)$

(d) Error $s(t)-s_{\text {rec }}(t)$

图 9 测得的 $F(t)=\Delta I_{\mathrm{ph}}(\mathrm{t}) / I_{\mathrm{ph} 0}$ 重构 $s(t)$ 波形。对于 $2 \mu \mathrm{m}$ 峰一峰信号, 误差 $< \pm 2 \mathrm{~nm}$ 。(见文献 $[21]$ )

Fig. 9 Reconstruction of the $s(t)$ waveform from the measured $F(t)=\Delta I_{\mathrm{ph}}(\mathrm{t}) / I_{\mathrm{ph} 0}$. For a $2 \mu \mathrm{m}$ peak-to-peak signal, error keeps $< \pm 2 \mathrm{~nm}$. (see Ref. [21])

当信号振幅较大时可得到类似的结果, 即 $N$ 倍的波长 $\lambda$ : 当 $N$ 达到 $10 \sim 20$ 时, 误差 $<5 \sim 10$ $\mathrm{nm}$, 然而 $N>100$ 时, $C$ 因子以及 $\alpha$ 因子较小的误 差就可能导致重构时产生 $\pi$ 相位 (或 $\lambda / 2$ 振幅) 的误差。
G. Plantier 等人提出了一种更复杂的基于成 本函数最小化算法 ${ }^{[22]}$, 该成本函数描述了测量数 据的距离二次方关系, 该数据是对参考波形进行 理论计算而获得的, 参数 $C$ 和 $\alpha$ 可调整。最小化 的误差给出了 $C$ 和 $\alpha$ 的最佳估算值, 且用波形直 
接分析法也发现了自洽性估算确保了其准确度。

综上所述,尽管半条纹技术包含了 $\mathrm{mm}$ 或亚 $\mathrm{mm}$ 振幅,但上述算法中没有一种可实时重构大 幅度 (即到 $m m$ 或 $\mathrm{cm}$ 范围) 的位移。

\section{3 正弦/余弦重构}

适用于所有干涉仪且对大振幅位移或振动信 号能够解决去包裹或重构问题有效的方法是基于 对 $\cos 2 k s$ 和 $\sin 2 k s$ 两个正交信号的处理。它们 是在双通道激光干涉仪 ${ }^{[5,7]}$ 中所寻求的用于数字 或模拟处理的两个信号,也是对 $C \ll 1$ 的 SMI 希 望能获得的。对于激光二极管 SMI, 因为是对光 学频率的 FM 驱动, 无法直接操纵电信号, 所以无 法得到 $\sin 2 \boldsymbol{k} s$ 信号。但是, 如果采用双模工作的 激光源, 一个模用于 SMI, 另一个在腔里保持模不

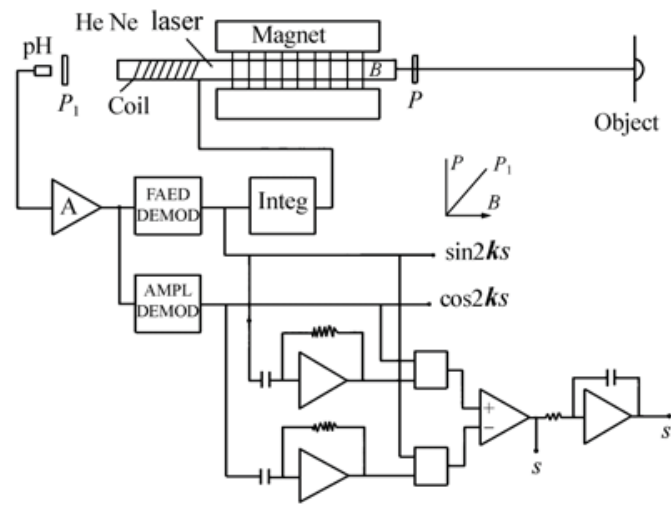

(a)

变作为解调器操作的区域振荡器, 引人电频率的 FM 调制,可获得用于重构的 $\sin 2 \boldsymbol{k} s$ 。

这是早在 1977 年证明的自混合干涉仪重构 技术 ${ }^{[7]}$, 也是第一篇报道的 SMI 可用于位移重构 的文章。为了实现这一目标, 采用 $\mathrm{He}-\mathrm{Ne}$ 塞曼激 光器 ${ }^{[5]}$, 其激活介质被加在介质上的磁场分为两 部分。S. Zhang 及其同事采用过该方法 ${ }^{[26-27]}$ 。在 横向塞曼效应下 ${ }^{[5]}, \mathrm{He}-\mathrm{Ne}$ 激光器支持具有线性 正交偏振的双模, 其频差为 $20 \sim 100 \mathrm{kHz}^{[5,7]}$ 。将 其中一个模式到达目标 (见图 10(b)) 而另一个 到达后面的光电探测器, 并用于将 SMI 信号从光 频转换到电频。光电探测器输出端的信号表现出 振幅的小起伏 $\mathrm{AM}, \mathrm{FM}$ 为正弦颤动。 $\mathrm{AM}$ 和 $\mathrm{FM}$ 解调后, 可获得两个信号 $S=\sin 2 \boldsymbol{k} s$ 和 $C=\cos 2 \boldsymbol{k} s$,

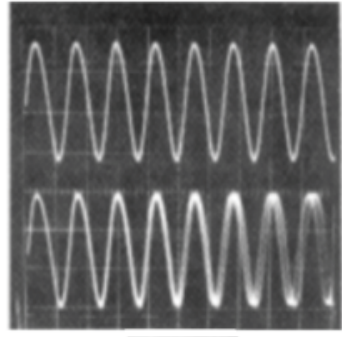

$20 \mu \mathrm{s}$

(b)

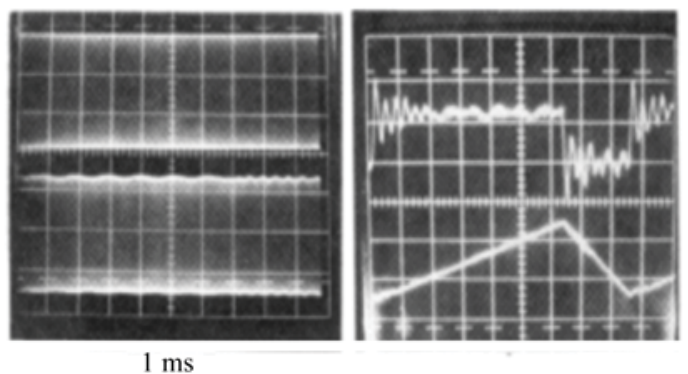

(c)

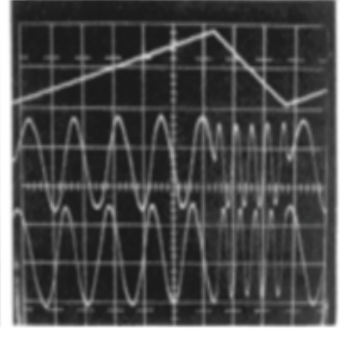

图 10 (a) 用 $\cos / \sin$ 信号重构位移: (b) 利用具有横向塞曼效应的 $\mathrm{He}-\mathrm{Ne}$ 激光器产生一对正交的、线性偏振模, 在 目标输出端放置一个偏振片, 选择出一个模用于自混合效应, 而另一模在腔内不受影响。在后反射镜位置 放置一 $45^{\circ}$ 偏振片使得两模输出 (一个载有 SMI 信号, 另一个为固定参考信号) 到光电探测器。(c) 振幅和 频率解调后给出 $\cos 2 \boldsymbol{k} s$ 和 $\sin 2 \boldsymbol{k} s$ 信号, 然后交叉相乘信号和其导数得到 $v$, 再对 $v$ 积分得到 $s$ ( 见文献 [7] )。

Fig. 10 ( a Reconstruction of displacement with the cos/sin signals : ( b ) a HeNe laser with transverse Zeeman effect is used to create a pair of orthogonal, linear-polarized modes. A polarizer at the target output selects one mode for selfmixing effect, while the other is kept unaffected in the cavity. A $45^{\circ}$ oriented polarizer at rear mirror allows beating of the two modes (one carrying the SMI signal, the other a fixed reference) on the photo-detector. (c) Amplitude and frequency demodulation reveals the $2 \boldsymbol{k s}$ and $2 \boldsymbol{k s}$ signals. The cross product of signals and their derivatives are made to free out $v$ and then integrate $v$ to obtain $s$ (see Ref. [7]) 
如图 10(c) 所示 ${ }^{[5,7]}$ 。对 $S$ 和 $C$ 的时间求导, 可得 到 $S^{\prime}=2 \boldsymbol{k} v \cos 2 \boldsymbol{k} s$ 和 $C^{\prime}=-2 \boldsymbol{k} v \sin 2 \boldsymbol{k} s, v=\mathrm{d} s / \mathrm{d} t$ 是 $s$ 的时间导数。 $C$ 和 $S$ 与其导数交叉相乘并相减, 得到:

$S^{\prime} C-S C^{\prime}=2 \boldsymbol{k} v \cos ^{2} 2 \boldsymbol{k} s+2 \boldsymbol{k} v \sin ^{2} 2 \boldsymbol{k} s=2 \boldsymbol{k} v$ 然后对 $v$ 积分得到 $s$ 。

$v$ 和 $s$ 的重构波形与电驱动波形相比有小的 波动, 但这仅仅是由实验中所用的换能器 (扩音 器) 的频率响应引起的, 即是对换能器的测量。

重构计算没有动态范围的上限, 因此可对任 意振幅进行计算, 如果选择替代模拟电路, 则用 PC 中的 ADC 获取信号可使处理过程数字化。因 为很难得到两个较为稳定且容易分离的频差很小 的正交模, 因此用激光二极管不能复制同一结构。

\section{4 速度测量}

根据是否测量 $v$ 的纵向分量, 即平行于波矢 量 $\boldsymbol{k}$ (或平行于视线) 的 $v_{/ / k}$, 或横向矢量 $v_{\perp k}$ (垂 直于波矢 $\boldsymbol{k}$ 或视线),主要有两种形式的 SMI 测 速计。

在第一种情况下, 相位 $2 \boldsymbol{k} s$ 表示为 $\phi=2 \boldsymbol{k}$. $s$ 。速度 $v=\mathrm{d} s / \mathrm{d} t$, 通过 $\phi$ 对时间求导, 有 $(\mathrm{d} / \mathrm{d} t)$ $2 \boldsymbol{k} \cdot s=2 \boldsymbol{k} \cdot v=\mathrm{d} \phi / \mathrm{d} t=\omega$ 。这表明 SMI 输出信号 $I_{\mathrm{ph}}$ 已经包括了由波失 $\boldsymbol{k}$ 即 $\boldsymbol{k} \cdot v=v_{k}$ 确定的沿视 线方向的速度分量, 速度 $v_{k}$ 可由信号 $I_{\mathrm{ph}}$ 中的频率 $f=\omega / 2 \pi$ 而测得, 尺度因子为 $2 \boldsymbol{k} / 2 \pi=2 / \lambda, v_{k}$ 有 时被称作多普勒信号, 但是它没有比干涉相移更 多的信息。所以,纵向速度的测量仅仅是 SMI 工 作原理的一个副产品, Scalise 等人 ${ }^{[28]}$ 讨论了几个 变量。如果忽略横向分量, 运动过程中目标点不 变,因而散斑统计特性不影响测量。

在考虑横向速度分量时, 令 $2 \underline{k} \cdot \underline{v}=0$, 此时 不应该观察到 SMI 信号的任何输出,然而还是观 察到了一些与速度有关的现象, 因为当缺失干涉 信号时, 散斑统计特性影响激光器中的其它常数 场, 产生随机被调制的输出电流 $I_{\mathrm{ph}}$ 。 $I_{\mathrm{ph}}$ 的频率常 数可通过特别标定 ${ }^{[29-30]}$ 手段与速度分量 $v_{\perp k}$ 产生 相关关系,然而基于这一原理的速度计有时会由 于目标表面结构而出现不稳定现象。

\section{5 距离测量}

与其它干涉仪相同, SMI 基于相位检测原理, 所以需要乘以 $2 \pi$ (或乘以 $\lambda$ ) 来计量相位增量。
实际上, SMI 测量的不是到目标的距离, 而是以小 步长 (即 $\lambda / 2$ ) 为增量的累积位移, 为此需要从 $z=0$ 移动到 $z=s$ 后计量步长的增量。这不同于干

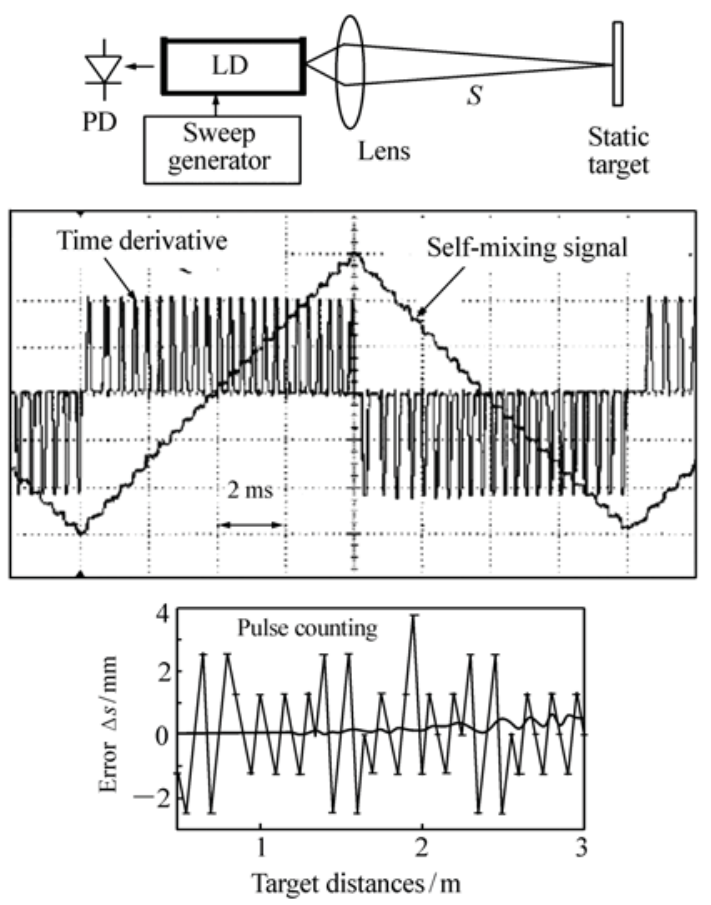

图 11 SMI 距离测量: 在激光二极管上用偏置电流 扫描, 用一个三角波调制波长, 相位 $\phi=2 k s$ 表现出 $N$ 个 $2 \pi$ 周期变化 (对应波形上的小 起伏)。SMI 信号以时间微分和周期 $N$ 计数, 距离单位尺度是 $\lambda^{2} / 2 \boldsymbol{k} \Delta \lambda$, 所以距离 $s=$ $N \lambda^{2} / 2 \boldsymbol{k} \Delta \lambda$ 。下图是 $s=1 \sim 3 \mathrm{~m}$ 的距离测量 (见文献 $[3]$ )

Fig. 11 Distance measurement with a SMI : by a bias current sweep applied to the laser diode, wavelength is modulated with a triangular waveform, and phase $\phi=2 \boldsymbol{k} s$ exhibits a number $N$ of $2 \pi$-periods variations (the small ripple on waveform). The SMI signal is time-differentiated and the periods $N$ counted. Unit of scale distance is $\lambda^{2} / 2 k \Delta \lambda$ and accordingly distance is $s=N \lambda^{2} / 2 k \Delta \lambda$. Diagram at bottom is the spread of measurements on a $s=1.3 \mathrm{~m}$ distance( see Ref. [3])

涉仪相位传感仪器进行的距离测量。为了克服这 一限制, Gouaux 等人 ${ }^{[31]}$ 提出了利用偏置电流 $I_{\text {bias }}$ 引起波长变化的关系从相位 $\phi=2 \boldsymbol{k} s$ (模为 $2 \pi$ ) 中 求出 $s$ 。为此, 对偏置电流进行线扫描, 得到幅值 
$\Delta I_{\text {bias }}$ (从最小的 $I_{0}$ 到最大 $I_{0}+\Delta I_{\text {bias }}$ ), 如图 11 所 示, 如果 $\alpha_{\lambda}=\mathrm{d} \lambda / \mathrm{d} I$ 是电流波长系数, 波长变化为 $\Delta \lambda=\alpha_{\lambda} \Delta I_{\mathrm{b}}$, 相应的波数 $\boldsymbol{k}$ 变化为 $\Delta \boldsymbol{k}=\left(2 \pi / \lambda^{2}\right)$ $\Delta \lambda^{[29,31-33]}$ 。对静态目标, SMI 的光学相移变量由 $\Delta \phi=2 s\left(2 \pi / \lambda^{2}\right) \Delta \lambda$ 给出, 其中 $s$ 为常数。除以 $2 \pi$, 得到 SMI 干涉信号的周期数 $N=2 s \Delta \lambda / \lambda^{2}$ 。 从这一等式中解得 $s$ 为:

$$
s=N \lambda^{2} / 2 \Delta \lambda .
$$

所以,相对增量而言, 绝对距离测量是通过扫 描偏置电流得到 $\Delta \lambda$, 然后计量在扫描时间内的自 混合信号的周期数 $N$, 再利用式 (9) ${ }^{[31-32]}$ 求出距 离 $s$ 。

考虑其分辨率, 由式 (9) 所示, 距离测量单位 是因子乘以 $N$, 或 $d_{\text {unit }}=\lambda^{2} / 2 \Delta \lambda$ 。所以要得到较

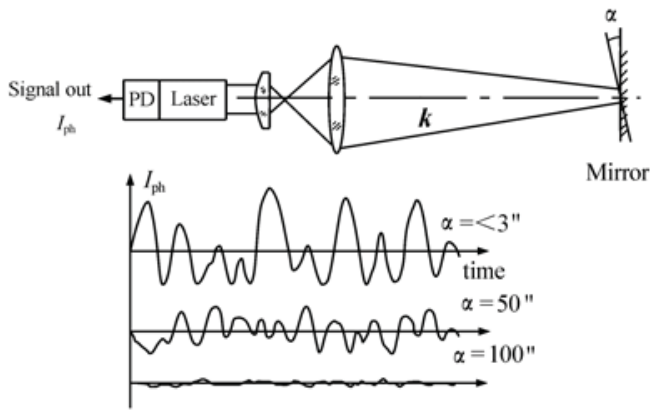

(a)
高的分辨率就需要较大的 $\Delta \lambda$ 。通常利用的法布 里-泊罗激光二极管因受到模式跳跃问题的限制, 则有 $\Delta \lambda=0.1 \mathrm{~nm}(\lambda=0.85 \mu \mathrm{m})$, 对应地有 $d_{\text {unit }}$ $=3.6 \mathrm{~mm}$ 。图 11 描述了由 SMI 距离测量仪器得 到的波形,并给出在 $1 \sim 3 \mathrm{~m}$ 距离重复性测量的例 子 $^{[3]}$ 。

\section{6 角度测量}

早期的 SMI 很容易探测从远距离目标反射 镜反射回的信号, 因为从周围收集到的麦克风产 生的振动就能够提供足够大的 SMI 信号 ${ }^{[34]}$ 。该 设备被改进后 ${ }^{[35]}$, 成为角度测量仪器, 其性能可 与光学自准直仪相比。该 SMI 装置 (见图 12) 利 用 PZT 驱动移动台, 移动台上安装激光二极管的 物镜以调整瞄准角 $\alpha$, 并得到响应信号 $I_{\mathrm{ph}}$ 。

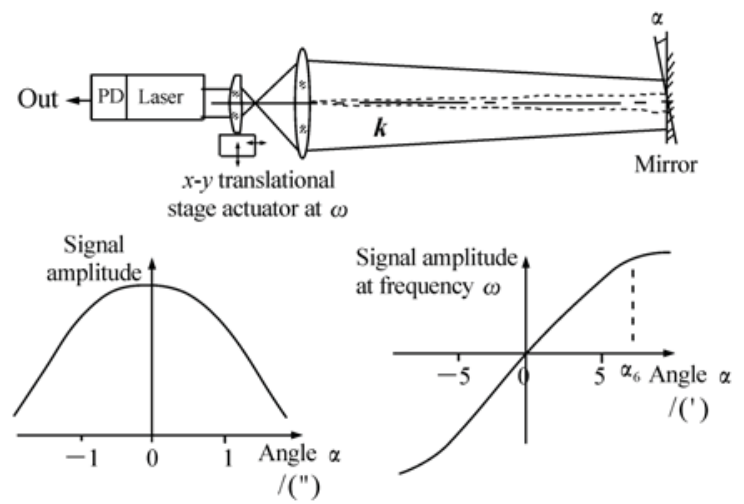

(b)

(c)

图 12 SMI 角度测量: 当远距离反射镜调整好后, SMI 信号由于周围的麦克风作用而被最大化 (a)。在改进的结构 中, 用一个 $X Y$ 方向压电驱动来调制物镜角度, 使得 SMI 信号可对驱动信号的振幅和相位进行传感 $(+/-$ 符号对应正/反相)。抛物线状曲线 (b) 被转换成过零点的准线性曲线 (c), 对应的动态范围从几弧分到零点 几弧秒 (见文献[35])

Fig. 12 Alignment and angle measurement with a SMI: when a remote mirror is well aligned, the SMI signal due to ambient microphonics is maximized(a). In an improved setup, the angle is modulated by an $X Y$ piezo actuator slightly moving the objective lens. The resulting SMI signal is sensed in amplitude and phase respect to the drive signal $(+/-$ sign for phase/antiphase $)$. The parabolic-like response curve $(b)$ is thus transformed in a quasi-linear passing through the zero(c). Angles down to a fraction of arc-sec can be measured on a dynamic range of a few $\operatorname{arc}-\min ($ see Ref. [35])

众所周知,一般仪器的使用均力图达到高精 度的最大响应条件,这里寻找 $I_{\mathrm{ph}}$ 信号的相位关系 并与压电陶瓷 (PZT) 驱动进行比较。将一般 SMI 信号对 $\alpha$ 近似地二次方响应关系等效地转换到 线性关系,最优的结构布置(图 12 (c) ) 是 $\alpha=0$ 条件下的过零点。实验表明:用传统的元件,噪音
限的分辨率约为 $0.2^{\prime \prime}$, 动态范围约为 $5^{[\text {[35] }}$ 。

\section{5 激光参数测量}

根据 SMI 信号波形和其细部特征可知它们 不是偶然的, 而是与光源的某种物理参数相关。 
特别是在 $C<1$ 时, 波形断点的起伏揭示了激光 器的线宽细节, 而这时的波形破坏和突变位置是 耦合强度参数 $C$ 、线宽增强因子 $\alpha$ 的特征。下面 将给出基于波形分析测量这两个参数的原理。

\section{1 激光线宽}

自混合信号中,陡峭下降突变的相位起伏反 映了如图 13(a) 所示的波形起伏, 该现象是由目 标位移 $s=L_{0}+\Delta L$ 中的小变化 $\Delta L$, 或由波矢围绕 $\boldsymbol{k}_{0}=2 \pi v_{0} / c$ 的变化 $\Delta \boldsymbol{k}=\Delta v / c$ 引起的。Giuliani 和 Norgia ${ }^{[36]}$ 认为两者的贡献之和作用于相位变 量 $\left\langle\phi^{2}\right\rangle$, 且可得出:

$$
\begin{gathered}
\Delta\left\langle\boldsymbol{\phi}^{2}\right\rangle=\Delta\left\langle\left(2 \boldsymbol{k}_{\mathrm{s}}\right)^{2}\right\rangle= \\
4\left[\boldsymbol{k}_{0}^{2}\left\langle\Delta L^{2}\right\rangle+L_{0}^{2}\left\langle\Delta \boldsymbol{k}^{2}\right\rangle\right]= \\
(4 \pi / c)^{2}\left[v_{0}^{2}\left\langle\Delta L^{2}\right\rangle+L_{0}^{2}\left\langle\Delta v^{2}\right\rangle\right] .
\end{gathered}
$$

$\Delta\left\langle\phi^{2}\right\rangle$, 并将其带人 $L_{0}^{2}\left\langle\Delta v^{2}\right\rangle+$ const, 其中常数项 为 $(4 \pi / c)^{2} v_{0}^{2}\left\langle\Delta L^{2}\right\rangle$ 。如图 13 所示 ${ }^{[36]}$, 在 $L_{0}=$ $10 \mathrm{~cm}$ 到 $3.5 \mathrm{~m}$ 的位置, 对后反射器施加一小 (几 个波长) 的驱动信号, 得到增量很小的常数项 $(c=0.1 \mathrm{rad})$ 。根据式 (10) 将二次项叠加, 通过 $L_{0}$ 以 $50 \mathrm{~cm}$ 步长增加几步就可很容易测定线宽 项 $L_{0}^{2}\left\langle\Delta v^{2}\right\rangle$ 。如图 13 所示, 从几米的位移就可测 得 $3.5 \sim 14 \mathrm{MHz}$ 的线宽 $\Delta v$, 最小可测线宽约为 $1 \mathrm{MHz}_{\circ}$ 。由这一极限可知, 该方法需要较小的实 验空间, 只要能满足基于不匹配 (延迟外差) 的测 量方法所需的 $1 \mathrm{MHz}$ 时的传播长度 $c / \Delta v \approx 300 \mathrm{~m}$ 即可。

该方法还给出了相干长度: $L_{\mathrm{c}}=c / \sqrt{\left\langle\Delta v^{2}\right\rangle}$, 即需要外部臂长 $L_{0}$ 远小于相干长度 $L_{\mathrm{c}}$ 。

通过齿状驱动作用 $\Delta L$, 测量整个相位变化

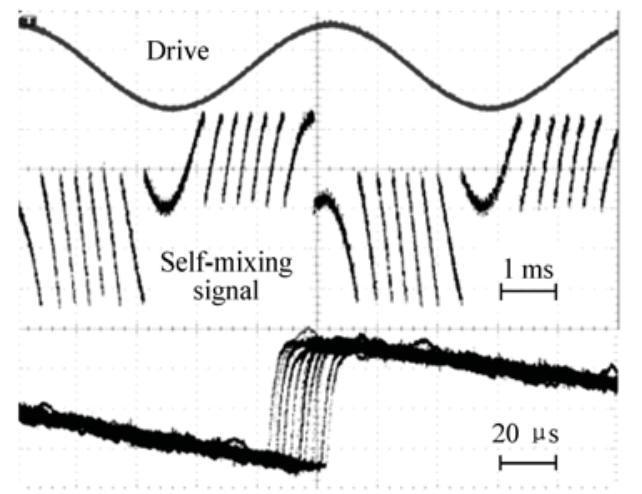

(a)

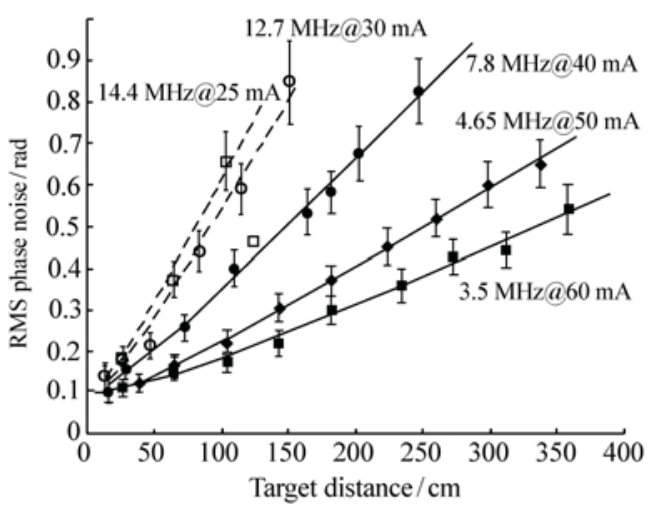

(b)

图 13 (a) 利用 SMI 进行线宽测量: 激光器处于 $C>1$ 状态, 寻找在自混合波形上出现突变时的颤抖。相关的相位 起伏变化为 $\Delta 2 \boldsymbol{k} s=2 \boldsymbol{k} \Delta s+2 s \Delta \boldsymbol{k}$, 其中第一项保持不变, 第二项 $2 s \Delta v / c$ 正比于线宽 $\Delta v$ 。 (b) 通过拟合变化量 的平方和得到不同激光器的线宽。( 见文献 $[36]$ )

Fig. 13 Line width measurement by SMI : the laser is set to a $C>1$ regime, then the jitter of the switching transient appearing in the self-mixing waveform is searched (a). The variance of the associated phase fluctuation $\Delta 2 \boldsymbol{k} s=2 \boldsymbol{k} \Delta s+2 s \Delta \boldsymbol{k}$, has a first term which is kept constant, and a second term $2 s \Delta v / c$ proportional to the line width $\Delta v$. The quadratic sum of variance contributions is fitted to obtain the line widths of different laser specimens(b). (see Ref. [36])

\section{$5.2 \alpha$ 因子测量}

SMI 信号的波形可表达为 $F(\phi)=\cos \omega \tau$ 和 式(3)。波形形状取决于反馈因子 $C$ 和线宽增强 因子 $\alpha$, 可用 SMI 信号得到这两个因子。2004 年, $\mathrm{Y} . \mathrm{Yu}$ 等人 ${ }^{[37]}$ 提出了基于 $\mathrm{SMI}$ 的 $\alpha$ 因子测量 方法,其中的 SMI 工作在中等光学反馈区。为说 明该方法, 先在式 (3) 中引人 $\phi=\omega \tau, \phi_{0}=\omega_{0} \tau$, 所
以:

$$
\phi=\phi_{0}-C \cdot \sin [\phi+\arctan (\alpha)] .
$$

图 14 给出了一个 SMI 波形的例子, 其中 $C=$ $2, \alpha=5$ 。 $\phi_{0}$ 增加时, $F(\phi)$ 沿路径 $A_{1}-B-B_{1}$ 变 化, 若 $\phi_{0}$ 减少, 路径则为 $B_{1}-A-A_{1}$ 。面 $A_{1}-B-$ $B_{1}-A$ 被称作迟滞面。相位延迟 $\phi_{0 . C B}$ 和 $\phi_{0 . A D}$ 可 从式(11) 中获得 ${ }^{[37]}$ : 


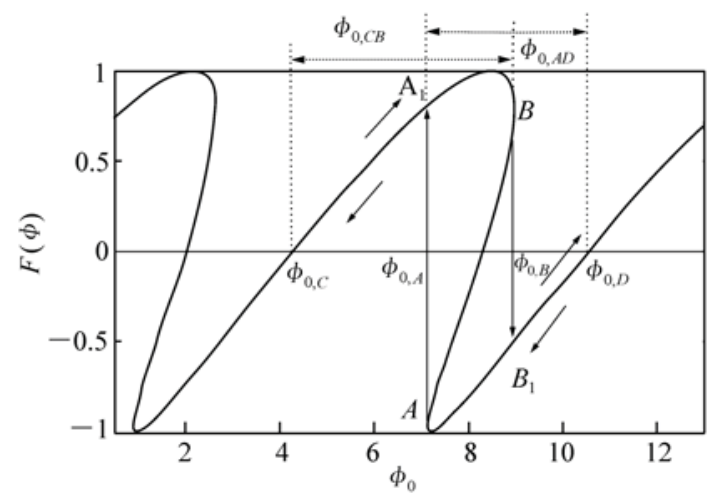

图 $14 C=2$ 和 $\alpha=5$ 时 SMI 信号的迟滞。寻找过零 点 $\phi_{0 . C}$ 和 $\phi_{0 . D}$ 以及突变点 $\phi_{0 . A}$ 和 $\phi_{0 . B}$, 可确定 $\alpha$ 因子(见文献 [37])

Fig. 14 Hysteresis of SMI signal with $C=2$ and $\alpha=5$. Looking at the zero-crossing points $\phi_{0 . C}$ and $\phi_{0 . D}$ and at the switching points $\phi_{0 . A}$ and $\phi_{0 . B}$, the $\alpha$-factor can be determined ( see Ref. [37]).

$$
\begin{gathered}
\phi_{0 . C B}=\sqrt{\left(C^{2}-1\right)}+C / \sqrt{\left(1+\alpha^{2}\right)}+ \\
\arccos (-1 / C)-\arctan \alpha+\pi / 2 \\
\phi_{0 . A D}=\sqrt{\left(C^{2}-1\right)}-C / \sqrt{\left(1+\alpha^{2}\right)}+ \\
\arccos (-1 / C)+\arctan \alpha-\pi / 2 .
\end{gathered}
$$

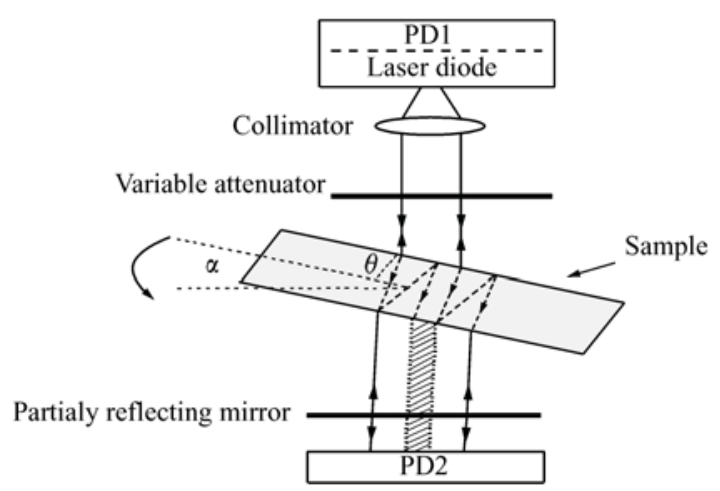

(a) $\phi_{0 . C B}$ 和 $\phi_{0 . A D}$ 可由图 14 中的 4 个特征点确 定, 即 2 个过零点和 2 个突变点。根据 SMI 波形 可测得两相位延迟, 所以可由式(12) 计算出 $C$ 和 $\alpha$ 。该方法给出了 $1<C<3.5$ 时的测量解。

当 $C$ 增加到一定程度时, 迟滞面积变宽, 这 使得过零点 $\phi_{0 . C}$ 和 $\phi_{0 . D}$ 被隐藏, 而在弱反馈条件 下 $(C<1)$ 突变点消失。两种情况下都可采用数 据滤波处理等方式得到 $C$ 和 $\alpha$, 如利用 SMI 理论 模型算法对 $\alpha$ 和 $C$ 进行优化, 估算得到与实验信 号最佳的匹配值 ${ }^{[38-39]}$ 。与相位方法 (4) ${ }^{[37]}$ 不同, 为了以较高的精度估算 $\alpha$, 需对整个 SMI 波形进 行数据滤波 ${ }^{[38-39]}$ 。

上述方法基于 SMI 时域波形分析。由于特 殊波形可能对测量有些限制, Y. Yu ${ }^{[40]}$ 等人提出 一种精度改善的在频域分析 SMI 信号从而测量 $C$ 的方法,该方法对高光学反馈水平也适用。

\section{6 物理量测量}

下面介绍几个用 SMI 测量物理量的方法, 这 些方法主要利用了 SMI 装置简单等特点。

\section{1 厚度和折射率}

测量透明平板厚度最常用的仪器是剪切干涉

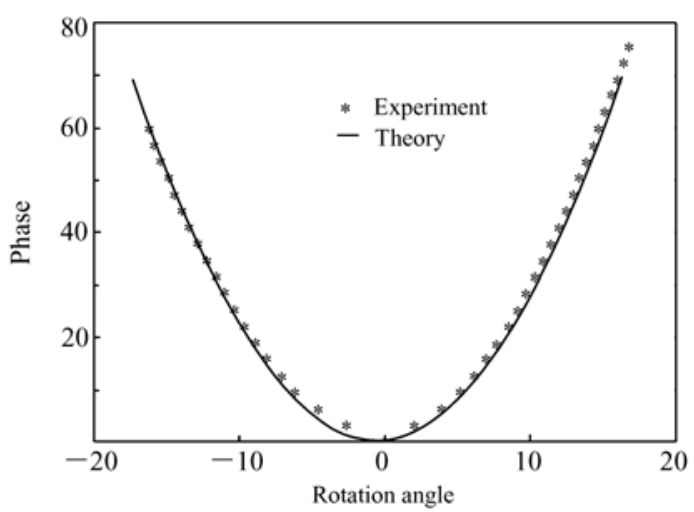

(b)

图 15 用 SMI 测量透明平板厚度: (a) 激光二极管作为自混合传感器 (为光二极管 $\mathrm{PD}_{1}$ 输出) 同时也是横向剪切干 涉仪的光源 (由光二极管 $\mathrm{PD}_{2}$ 输出); (b) 两条输出条纹相减给出 $\boldsymbol{k} d \cos \alpha$, 它是倾角 $\alpha$ 的函数,与 $n$ 无关。可 测厚度约为 $5 \sim 1000 \mu \mathrm{m}$ ( 见文献 $[42]$ )

Fig. 15 Transparent slab thickness measurement with SMI: a laser diode acts as a self-mixing sensor( output on photodiode $\mathrm{PD}_{1}$ ) and as a source of a lateral shear interferometer( output on photodiode $\left.\mathrm{PD}_{2}\right)(\mathrm{a})$. Subtraction of the fringes of the two outputs gives $\boldsymbol{k} d \cos \alpha(\mathrm{b})$, and it is the function of tilt angle $\alpha$ and independent from $n$. Range of thickness is measured about from 5 to $1000 \mu \mathrm{m}$ ( see Ref. [42]) 
仪, 激光器出射光与在平板两面反射回来的光叠 加在光电探测器 $\left(\mathrm{PD}_{2}\right)$ 上 (见图 $15(\mathrm{a})$ ), 产生的 干涉信号类型为:

$$
\Delta \phi_{\mathrm{PD}_{2}}=2 \boldsymbol{k} n d \cos \theta .
$$

与光程测量相同, 相位与厚度 $d$ 和平板折射 率 $n$ 有关。在 $\mathrm{PD}_{1}$ 中也可得到另外一路干涉信号 (见图 15 ), 该信号由通过平板的光束到光电二极 管 $\mathrm{PD}_{2}$, 然后返回到光源而产生。在光电二极管 $\mathrm{PD}_{1}$ 上产生第二个 $\mathrm{SMI}$ 相位信号:

$$
\Delta \phi_{\mathrm{PD}_{1}}=2 \boldsymbol{k} d(n \cos \theta-\cos \alpha) .
$$

将式(13)和(14) 相减, 可得到 ${ }^{[41]}$ :

$$
\Delta \phi=\Delta \phi_{\mathrm{PD}_{2}}-\Delta \phi_{\mathrm{PD}_{1}}=2 \boldsymbol{k} d \cos \alpha .
$$

该结果不再与 $n$ 有关。以 $\alpha \in-30 \sim+30^{\circ}$ 旋转平板测量剪切和 SMI 信号, 调整尺度后计算 $\Delta \phi$, 由式 (15) 解得 $d$, 其单位是 $2 \pi / 2 \boldsymbol{k}$ 。可测厚 度一般是 $5 \sim 2000 \mu \mathrm{m}$, 精确度约为 $2 \%{ }^{[42]}$ 。测 得 $d$ 后再回到 $\mathrm{PD}_{2}$ 信号, 通过式 (13) 求解折射 率。

\section{2 机械共振}

在某些 SMI 测量中与 MEMS 中检测机械共 振相似,利用已知驱动波形 $s(t)$, 能大大简化信 号处理过程。

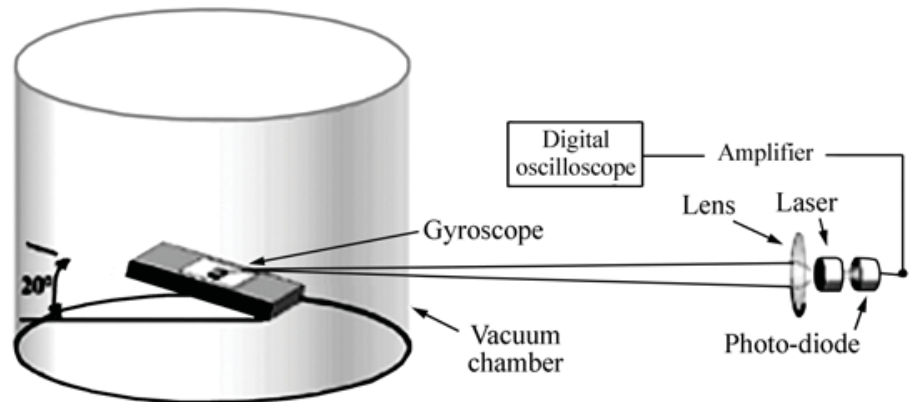

(a)
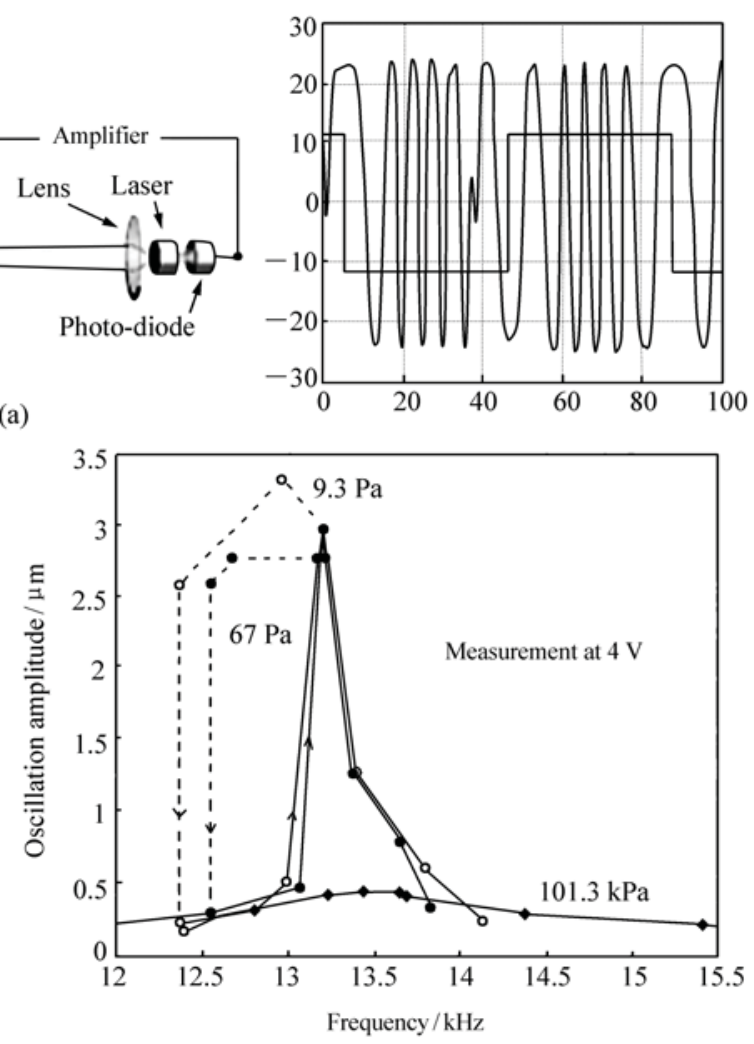

(c)

图 16 用 SMI 测试 $\mathrm{Si}$ 加工的 MEMS 机械性能。(a) 从激光器出射的光通过真空室的玻璃壁被会聚到芯片上的小振 动块上。以一定角度 $\left(\approx 20^{\circ}\right)$ 观察块的振动, 对 SMI 条纹信号进行恰当的修正。(b) 随着驱动电压的增加, 发现在 $8 \sim 9 \mathrm{~V}$ 时表现出共振频率漂移和随后的迟滞,表明初始的疲劳和爬行。(c) 因为残余空气摩擦共振 的 $Q$ 因子减弱,腔内压力增加。(见文献 $[43]$ )

Fig. 16 Test of the mechanical properties of Si-machined MEMS with SMI. (a) Light from the laser is focused on the small vibrating mass of the chip through the glass wall of a vacuum chamber. The vibration of the mass is viewed at an angle $\left(\approx 20^{\circ}\right)$, and the appropriate correction is applied to the SMI fringe signal. (b) As drive voltage is increased, a drift of resonant frequency can be found and then incipient hysteresis shows up at $8 \sim 9 \mathrm{~V}$, indicating incipient fatigue and creep. (c) At increasing chamber pressure, the $Q$-factor of resonance is damped because of residual air friction( see Ref. [43]). 
如图 16 所示, 用 SMI 激光束以一定倾斜角 (约 $20^{\circ}$ ) 瞄准测量一个陀螺仪 MEMS $^{[43-44]}$ 结构中 具有悬挂弹簧的测量块的面内运动, 可得到较大 的 $2 \boldsymbol{k} \cdot s$ 。典型测试块是一方形的硅板, 边长是 零点几个毫米, 板上有许多为减轻重量而刻蚀的 孔, 以确保高的共振频率。块表面表现出光学粗 粘性,SMI 能适用于这种不易测量的情况, 因为它 对散射目标适应性较强, 不受光点落在静止部分 或目标外面的影响,真空室的墙壁 (未经光学抛 光)对波前的破坏也不是问题。为了测量机械共 振, 用一方波激励 $V_{\mathrm{exc}}$ 产生梳状结构信号驱动 MEMS, 测量对应的位移幅度。

如果知道 $V_{\text {exc }}$ 起始时刻, 就很容易计数干涉 仪信号周期数, 即以步长 $\lambda / 2$ 测量其幅度。图 16 为以驱动电压 $V_{\text {exc }}$ 为参数的 MEMS( 图 16) 频率响 应图, 响应曲线迟滞揭示了初期疲劳和机械结构 的爬行情况, 给出了 MEMS 设计中的关键因素, 硅是常见的电子材料, 但其机械特性却被忽视, 特 别是高压条件下的机械特性。同样的测量,作为 压力的函数, 揭示了机械量中的实际阻尼因子, 这 个参数不易计算。

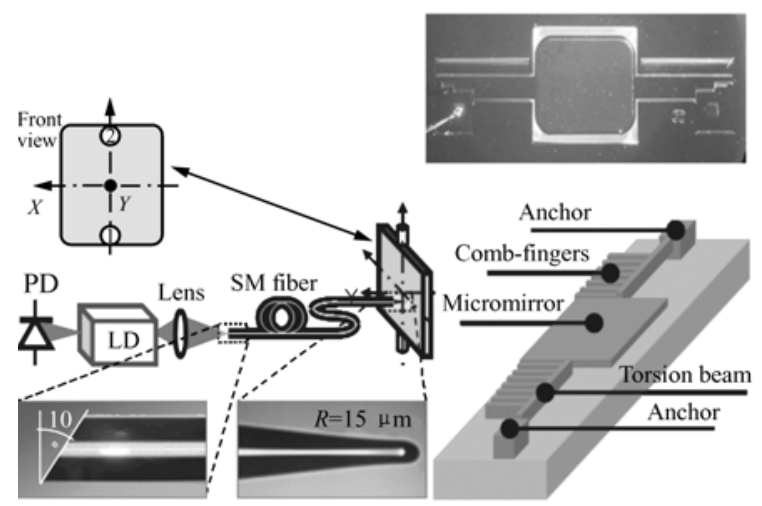

图 $17 \mathrm{Si}$ 基底上加工的 MEMS, 用单模光纤作为探 针对准微型反射镜进行测试, 与激光器耦合 的光纤端面有一定斜度, 而在传感反射镜运 动的一端逐渐变细形成透镜( 见文献 $[44]$ )

Fig. 17 In a Si-machined MEMS, micro-mirrors are tested by using a single mode fiber as the probe pinpointing the mirror. The fiber has a tilted splice at the laser coupling end, and is tapered and lensed at the other end to sense the mirror movement (see Ref. [44]).
对于 MEMS 结构的另一参数, V. Annovazzi 等 人 $^{[44]}$ 完成了光电开关微反射镜的测量。在 SMI 结构中插人一光纤尾纤(见图 17), 在目标端会聚 成极其微小的点到微型反射镜上, 从而探测激励 的响应和共振频率。

\section{3 应力/应变图}

在 3.2.1 节中, SMI 测振仪通过增加不同的 操作模式得到了进一步的发展, 可以测量叠加到 大的共模运动上的微小振动。

为了用传统的干涉仪进行差分测量, 通常可 以利用参考臂测量 $\phi_{1}-\phi_{2}=2 \boldsymbol{k}\left(s_{1}-s_{2}\right)$ 。然而， 如果在散射目标表面进行测量, 散斑统计可能削 弱相位差,并增加一个很大的误差相位项 $\phi_{\mathrm{sp}}$ 。用 带有反馈环路的 SMI 测振仪, 并对其内部相位信 号进行半条纹稳定, 可以消除误差项 $\phi_{\mathrm{sp}}$, 但是它 还缺少用于差分测量的第二个光学参考臂。也可 以减去电信号, 并且设置一个双通道 SMI 测振仪 来测量, 其中一个通道用于共模信号 $s_{\mathrm{CM}}$, 另一个 用于 $s_{\mathrm{CM}}+s_{\mathrm{D}}$, 由于包含了差分信号 $s_{\mathrm{D}}$, 因此可以 构建具有相同性能的两个通道 (响应不匹配率< $0.1 \%$, 噪声平台和动态范围差 $<5 \%)^{[45]}$ 。电子 相减差分和光学相位差分干涉仪的效果相当,都 可用于机械测试。

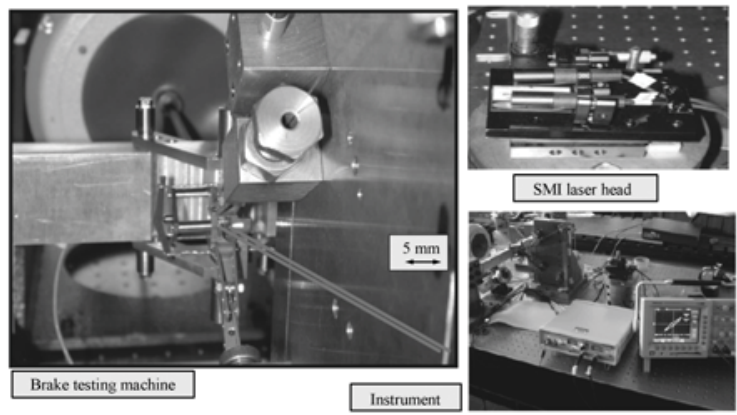

图 18 半条纹稳定的 SMI 测振仪可以差分模式测量 散射目标, 这里为对一个汽轮机马达制动的 消音珠子进行的测试。两束光照到摇动体 (共模) 和珠体上 (测量)。

Fig. 18 SMI half-fringe stabilized vibrometer can also work in differential mode on a diffuser target, here the damper bead of a turbine motor brake. The two beams point at the base of the shaker (common mode) and the bead body ( measurement) 
图 18 为制动珠实验台,其摇动机构使珠子在 制动支撑上产生振动。这个压力是一个准正弦 $V_{\mathrm{ST}}$ 激励, 支撑的振动为 $S_{\mathrm{CM}}$, 珠子振动为 $S_{\mathrm{D}}$ 。共 模宽约为 $15 \sim 30 \mu \mathrm{m}$, 差为 $0.5 \sim 4 \mu \mathrm{m}$ 。从力学 观点来看, $V_{\mathrm{ST}}$ 激励正比于压力 $T, S_{\mathrm{D}}$ 正比于机械 样品的应力 $S$ 。

用电子差分 SMI 可以测得 ${ }^{[45]}$ 样品的迟滞 $T$ $S$ 图,这也是首次获得的信息。如图 19 所示, 在 中等压力下样品处于弹性或牛顿区, $S$ 和 $T$ 线性 相关且无迟滞。当达到一定阈值时,材料进人塑 性区, 且图形随着迟滞打开, 迟滞周期扩大, 直到 $T$ 有小的增长,样品解体。收集这些信息对于设 计和测试机械结构具有很高的价值。
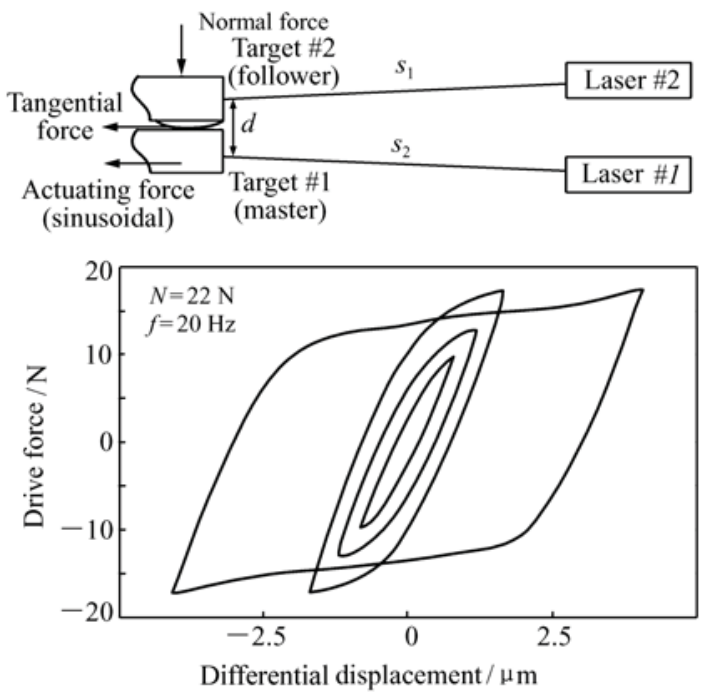

图 19 首次采用 SMI 差分测振计测得的应力应变 图, 揭示了牛顿区迟滞可以忽略 $(F<7 \mathrm{~N})$, 在塑性区迟滞环打开 $(F=8.15 \mathrm{~N})$, 珠子在解 体前释放能量

Fig. 19 Strain-stress diagram is measured optically for the first time with the SMI differential vibrometer, and reveals the Newtonian regime where hysteresis is negligible $(F<7 \mathrm{~N})$, and the plastic regime where the hysteresis loop opens $(F=8.15 \mathrm{~N})$ and the bead dissipates energy, before the breakdown occurs ( at $F \cong 17.5 \mathrm{~N}$ ).

\section{4 生物运行性}

正/余弦重构测振仪 (3.2.3 节) 已经被用于 获取生物信号 ${ }^{[46]}$ 。在心脏病的临床实验中,已经 观察到一个正常人指尖的心跳脉动,复制下心脏
脉动的形状, 比如 LFE (左心室排出) 和 DI (分色 c 切迹), 这些通常都可在正常心电图 (见图 20) 中观察到。

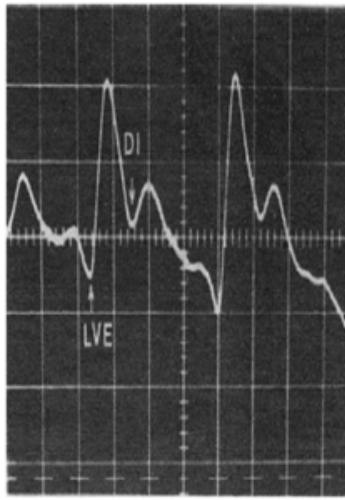

(a)

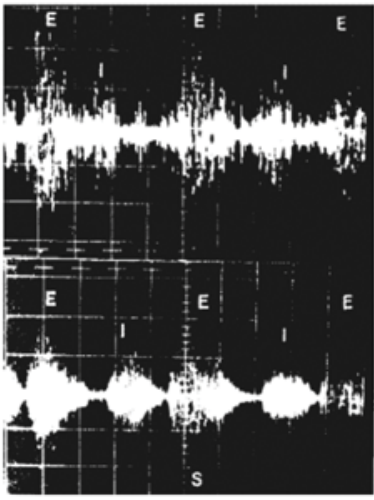

(b)
图 20 用 He-Ne SMI 测量生物信号, (a) 指尖的血流 脉冲 $(0.5 \mu \mathrm{m} / \operatorname{div}, 0.3 \mathrm{~s} / \operatorname{div}) ;(b)$ 在病人背 后探测的呼吸信号对比, 听诊器声音信号 (上) SMI 光学波形(下) (见文献 [3])

Fig. 20 Two samples of biomedical signals measured by the He-Ne SMI, (a) pulsation of blood on a finger tip $(0.5 \mu \mathrm{m} / \mathrm{div}, 0.3 \mathrm{~s} / \mathrm{div})$, (b) respiratory sounds detected on the back of a patient, with the acoustical signal of a stethoscope ( top ) compared to optical waveform taken by SMI( bottom) (see Ref. [3]).

J. Hast 等人 ${ }^{[47]}$ 使用另一种结构的 SMI 对 200 名志愿者进行了检测, 测量前臂径向动脉中 心血管脉冲的多普勒信号 $(\mathrm{d} \phi / \mathrm{d} t=2 \boldsymbol{k} v)$ 。检测 发现, $\mathrm{d} \phi / \mathrm{d} t$ 与从中指上测得的血压波形的时间 导数有很好的相关性 $(c=0.84)$, 证实了 SMI 做 出诊断结果的有效性。

正/余弦测振仪可以获得生物背部的呼吸 音 ${ }^{[46]}$ 。从测量到的呼气和吸气的声音( 见图 20 的 $\mathrm{I}$ 和 $\mathrm{E}$ ) 可以发现, 除了缺少波峰以外,波形和 传统听诊器得到十分相似, 而差别是由呼吸时听 诊器和皮肤的摩擦造成的。在从皮肤上获取运行 性信号的所有实验中, 皮肤表面都是未经处理的, 但患者必须被固定以避免因散斑图统计造成的 SMI 信号变坏。

最近,L. Rovati 等人报道了关于生物信号获 取的一个有趣的应用 ${ }^{[48]}$ 。用一个超发光二极管 
做 SMI 光源, 可以测量一个静脉模拟毛细管中的 液流的速度变化。A. Pesatori 等人 ${ }^{[49]}$ 报道了经 过改进的用于血流测量的 SMI, 该 SMI 中毛细管 以倾角 $\theta$ 与轴向对准, 从而得到信号 $2 \boldsymbol{k} \operatorname{scos} \theta$ 。R. Kliese 等人 ${ }^{[50]}$ 利用具有更短波长的 $\mathrm{GaN}(\lambda=$ $405 \mathrm{~nm}$ ) 和 $\theta=15^{\circ}$ 的倾斜度观察毛细血管,得到的 最小可测流速低达 $26 \mu \mathrm{m} / \mathrm{s}$ 。

\section{7 传 感}

考虑相位测量相反的情况, SMI 测量输人信 号的振幅时可用作传感器。如第 2 节所述, SMI 属于相干探测器类干涉仪 ${ }^{[5]}$, 它的输出与场幅度 成正比, 在探测量子限下被提取特征, 即 SNR 受 限于所进人光子流的量子化 (或粒度)。因此, SMI 具有高灵敏度感知小信号的能力,已被开发 应用到多种场合。

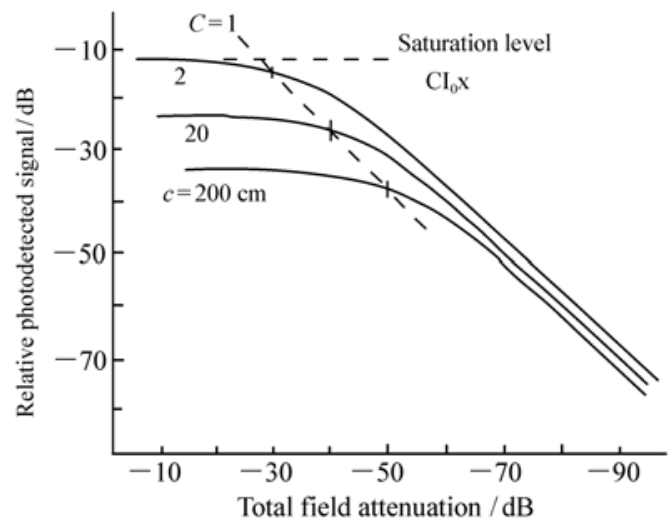

(a)

\section{1 远程回波和回波损耗}

对于远程回波的响应,SMI 很容易通过反射 镜和衰减器的组合让一部分出射能量重新进人激 光腔 ${ }^{[51]}$ 。对于一个典型的激光二极管 $(5 \mathrm{~mW}$, $\lambda=825 \mathrm{~nm}$ ), 探测信号的幅值与往返路径上的衰 减关系曲线如图 21(a) 所示。可以看出, SMI 可 以检测出 $10^{-9}$ 倍输出能量的回波, 由于其间注人 强区 $(C>1)$, 在约为 $10^{-3}$ 时发生饱和。因而回波 探测器是一个非常敏感的回波损耗测量仪 (见图 21 (c))。同时,它也可以对安装在激光芯片前面 的用于防止其受反射光影响的光学隔离器进行隔 离因子在线测量 ${ }^{[51-52]}$, 但测量时需要增加光路的 相位调制, 这样远程回波的振幅就从零频部分移 动至载波 $\omega_{0}$ (调制频率) 处, 避免了低频漂移, 更 便于振幅测量。相位调制器作为一个组件, 既可 以是以 PTZ 驱动的用于导波的光纤线圈, 也可以 是用于自由空间扩音的扬声器。

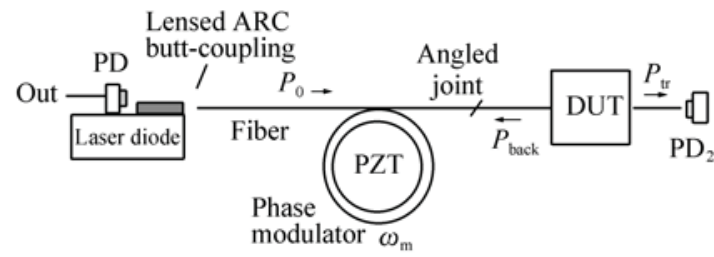

(b)

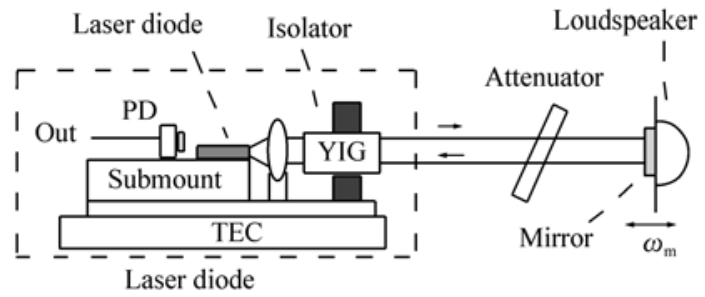

(c)

图 21 (a) 探测信号的幅值与往返路径上的衰减关系曲线。(b)作为回波探测器,SMI 可探测小至输出功率 $10^{-9}$ 的 信号。( c) 为了测量回波损耗或隔离损耗, 加一个载波频率 $\omega_{0}$ 、由绕在 PZT 上的线圈形成的相位调制器或 一个目标端的扩音器进行路径调制。SMI 信号以频率 $\omega_{0}$ 被读出, 它的幅值给出了 DUT 的回波损耗或插人 隔离器的损耗

Fig. 21 Relationship between total field attenuation and relative photodetected signal(a). As an echo detector, the SMI can sense returns as small as $10^{-9}$ of the output power(b). To measure the return loss or the isolation loss, a modulation of the path-length at a carrier frequency $\omega_{0}$ is added, through a coiled PZT phase-modulator or a target-end loudspeaker(c). The SMI signal output is then read at frequency $\omega_{0}$. Its amplitude provides the return loss of the DUT or the insertion loss of the isolator

图 21(右) 给出了两个测量方案: (1) 测量光 纤 DUT 的回波损耗; (2) 测量安装在芯片前的光
隔离器的隔离因子。这两种情况下, 返回能量的 灵敏性优于 $10^{-8}$ 或 $-80 \mathrm{~dB}^{[51]}$ 。 


\section{2 共焦显微镜}

SMI 传感器也已经应用于共焦显微镜中。根 据 C. -H. Lu et al. ${ }^{[53]}$ 的建议,使用 SMI 使得光路 调整过程更加简化( 见图 22)。
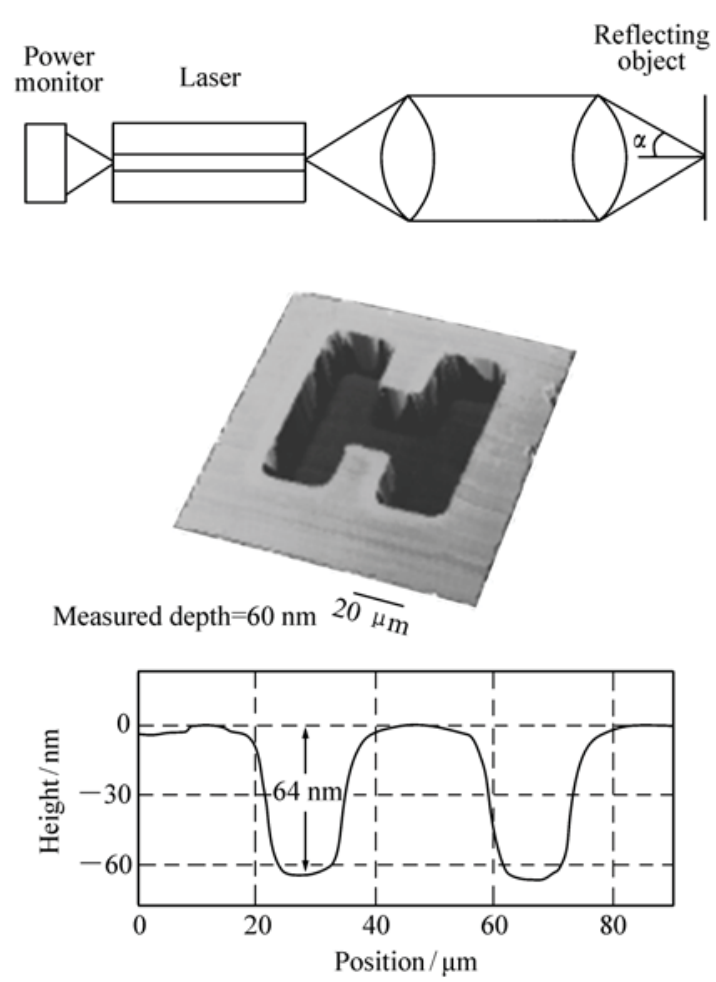

图 22 SMI 可被用在共焦显微镜中, 利用其自排列 探测器的优势对样件扫描, 得到具有空间分 辨率 $r=1 / \pi N A \approx 1 \mu \mathrm{m}$, 典型的深度分辨率为 $\Delta z \approx 30 \mathrm{~nm}$ 的图像 (见文献 [53])

Fig. 22 A SMI can be used in connection to a confocal microscope layout with the advantage of self-alignment of the detector. Scanning the specimen generates an image with a spatial resolution $r=1 / \pi N A \approx 1 \mu \mathrm{m}$ and a typical depth resolution $\Delta z \approx 30 \mathrm{~nm}$ ( see Ref. [53])

尽管受衍射限制的横向分辨率为 $\lambda / \pi \mathrm{NA}$,但 由于 SMI 对探测信号的电子处理, 纵向分辨率可
以达到 $30 \sim 50 \mathrm{~nm}^{[53]}$ 。 M. Wang 和 G. Lay 提 出 ${ }^{[54]}$ 了基于 SMI 共焦显微镜的扫描轮廓仪, 它对 边宽为 $5 \mu \mathrm{m}$ 的样品高度方向的分辨率达 $10 \mathrm{~nm}$ 。

\section{8 结束语}

本文给出了基于 SMI 而发展起来的各种仪 器概况。SMI 是一种基于激光二极管弱耦合现象 的测量方法, 不仅适用于传统干涉所涵盖的运动 学和尺度相关的测量, 而且也可用于物理参数的 测量,包括小信号探测, 回波探测等, 具有结构简 单、精度高等特点。

本文系统整理了 SMI 的测量领域,讨论了具 有不同特点 (如动态范围, 噪声, 周期/非周期位 移, 模拟/数字处理等) 的几种类型的 SMI, 它们性 能不同,代表了不同类型的仪器。位移测量时分 辨率可达到亚微米量级, 即使对光学特性不好的 目标, 其测量范围也可达到 $2 \mathrm{~m}$; 对于振动测量, 小振幅振动测量, 其最大动态范围为 $500 \mu \mathrm{m}$, 频 率可从 $0.1 \mathrm{~Hz}$ 到几十 $\mathrm{kHz}$ 。对于大振幅振动测 量, 振幅可达 $\mathrm{mm}$ 量级, 误差也在 $\mathrm{nm}$ 量级; 对速 度的测量, 虽然从原理上实现不是问题,但还存在 受目标表面特性影响等实际问题; 对距离的测量, 当测量距离在 $1 \sim 3 \mathrm{~m}$ 时, 其分辨率可达 $3.6 \mathrm{~mm}$; 对角度测量, 其性能可与高质量的光学自准直仪 相謧美; 在激光参数测量方面, 根据 SMI 波形和 其细部特征, 本文给出了耦合强度参数 $c$ 和线宽 增强因子 $\alpha$ 的测量原理。在远程回波或回波损 耗测量方面,目前的灵敏度已经达到 $-80 \mathrm{~dB}$; 在 物理量测量方面,已经实现了对透明平板厚度、折 射率、机械共振、应力应变的测量, 同时在生物运 行性和共焦显微镜方面, SMI 也获得开发和应用。

SMI 技术还远远没有得到充分的开发,在未 来的若干年中,它必将不断地为年轻的研究者提 供充分发挥创造力和才智的平台。

\section{参考文献：}

[1] SPENCER M B,LAMB W E. Laser with a transmitting window [J]. Phys. Rev. ,1972, A5:884-891.

[2] SPENCER M B ,LAMB W E. Laser with external injection[J]. Phys. Rev. ,1972, A5:891-897.

[3] DONATI S. Electrooptical Instrumentation [M]. Upper Saddle River, N. J. :Prentice Hall, 2004.

[4] LODI V A, DONATI S. Injection modulation in coupled laser oscillators [J]. IEEE J. Quantum Elect. ,1980,16:859865 . 
[5] DONATI S. Photodetectors [M]. Upper Saddle River, N. J. :Prentice Hall,2000.

[6] DONATI S, GIULIANI G, MERLO S. Laser diode feedback interferometer for the measurement of displacement without ambiguity[J]. IEEE J. Quantum Elect. ,1995(31):113-119.

[7] DONATI S. Laser interferometry by induced modulation of the cavity field [J]. J. Appl. Phys. ,1978,49:495-497.

[8] ACKET G A,LENSTRA D,DENBOEF A J, et al. . The influence of feedback intensity on longitudinal mode propeties and optical noise in index-guided semiconductor lasers[J]. IEEE J. Quantum Elect. ,1984(20) :1163-1169.

[9] RANDONE E, DONATI S. Self-mixing interferometer: analysis of the output signals [J]. Opt. Express, 2006, 14:97889796.

[10] WANG W M, GRATTAN K T W,PALMER A W , et al. . Self-mixing interference inside a single-mode laser diode for optical sensing applications[J]. IEEE J. Lightwave Techn. ,1994,12:1577-1587.

[11] PETERMANN K. Laser Diode Modulation and Noise[M]. Dodrecht:Kluwer Academic Publ. ,1991.

[12] LANG R, KOBAYASHI K. External optical feedback effects on semiconductor injection laser properties [J]. IEEE J. Quantum. Elect. ,1980,16:347-355.

[13] KANE D M K,SHORE K A. Unlocking Dynamical Diversity: Optical Feedback Effects on Semiconductor Lasers[ M ]. London: John Wiley \& Sons ltd,2008.

[14] HENRY C H. Theory of spontaneous noise in open resonators and its application to laser and optical amplifiers [J]. IEEE J. Lightwave Techn. ,1986(4) :288-1297.

[15] RUDE A F, WARD M J. Laser transducer system for high accuracy machine positioning[J]. Hewlett. Packard J. ,1976: $2-6$.

[16] DONATI S, FALZONI L, MERLO S. A PC-interfaced, compact laser-diode feedback interferometer for displacement measurements[J]. IEEE Trans. Instrument. Measur. ,1996,45:942-947.

[17] VODHANEL R S, KRAIN M, WAGNER R E. Long-term wavelength drift of the order of $-0.01 \mathrm{~nm} / \mathrm{yr}$ for 15 free-running DFB lasers modules[C]//OFC-94 Techn. Digest,San Jose,USA Feb. 20-25,1994.

[18] DONATI S, MARTINI G. Speckle-pattern intensity and phase second-order conditional statistics [J]. J. Opt. Society Am. , 1979,69:1690-1694.

[19] NORGIA M, DONATI S, D' ALESSANDRO D. Interferometric measurements of displacement on a diffusing target by a speckle-tracking technique[J]. IEEE J. Quantum Elect. ,2001,37:800-806.

[20 ] DONATI S. Laser interferometer[EB/OL] [2011-12-15]. http://www-3. unipv. it/donati/SM_Interfer_UniPV.pdf.

[21] MERLO S,DONATI S. Reconstruction of displacement waveform with a single-channel laser-diode feedback interferometer[J]. IEEE J. Quantum. Elect. ,1995,31:113-119.

[22] PLANTIER G, BES C, BOSCH T. Behavioral model of a self-mixing laser diode sensor[J]. IEEE J. Quantum. Elect. , $2005,41: 1157-1167$.

[23 ] GIULIANI G,BOZZI-PIETRA S,DONATI S. Self-mixing laser diode vibrometer[J]. Meas. Sci. Technol. ,2003,41:2432.

[24] DONATI S. Laser vibrometer[EB/OL] [2011-12-15]. http://www-3. unipv. it/donati/SMVibro_UniPV. pdf.

[25] BOASHASH B. Estimating and interpreting the instantaneous frequency of a signal-part I [ J ]. Proc. IEEE, 1992,80 (4) :519-538.

[26] MAO W,ZHANG S, CUI L, et al. . Self-mixing interference effects with a folding feedback cavity in Zeeman-birefringence dual frequency laser[[J]. Opt. Express, 2006,14:182-189.

[27] LIU G,ZHANG S, XU T, et al. . Self-mixing interference in Zeeman-birefringent dual frequency laser[J]. Opt. Commun. ,2004,241:159-166.

[28 ] SCALISE L, YU Y, GIULIANI G, et al. . Self-mixing laser diode velocimetry : application to vibration and velocity measurement[J]. IEEE Trans. Instrum Measur. ,2004,53:223-229.

[29] GIULIANI G, NORGIA M,DONATI S, et al. . Laser diode self-mixing techniques for sensing applications [J]. Optics A Pure Appl. Opt. ,2002, 4 :283-S294. 
[30 ] HAN D, WANG M,ZHOU J. Self-mixing speckle in an erbium-doped fiber ring laser and its application to velocity sensing[J]. IEEE Photonic Tech. L. ,2007,19:1398-1400.

[31] GOUAUX F,SARVAGENT N, BOSCH T. Absolute distance measurement with an optical feedback interferometer [J]. Appl. Optics, $1998,37: 6684-6689$.

[32] NORGIA M, GIULIANI G,DONATI S. Absolute distance measurement with improved accuracy using laser diode self mixing interferometry in a closed loop[J]. IEEE Trans Instrum Measur, 2007,56:1894-1900.

[33] GUO D, WANG M. Self-mixing interferometry based on a double-modulation technique for absolute distance measurement [J]. Appl. Optics, $2007,46: 1486-1491$.

[34] MATSUMOTO H. Alignment of length-measuring IR laser interferometer using laser feedback[ [J]. Appl. Optics, 1980, 19:1-2.

[35] GIULIANI G, DONATI S, PASSERINI M, et al. . Angle measurement by injection detection in a laser diode [J]. Opt. Eng. ,2001, 40:95-99.

[36] GIULIANI G, NORGIA M. Laser diode linewidth measurement by means of self-mixing interferometry[J]. IEEE Photonic Tech. L. ,2000,12:1028-1030.

[37] YU Y, GIULIANI G, DONATI S. Measurement of the linewidth enhancement factor of semiconductor lasers based on the optical feedback self-mixing effect[J]. IEEE Photonic Tech. L. ,2004,16:990-992.

[38 ] XI J, YU Y, CHICHARO J, et al. . Estimating the parameters of semiconductor lasers based on weak optical feedback interferometry [J]. IEEE J. Quantum Elect. ,2005,41(8):1058-1064.

[39] YU Y,XI J, CHICHARO J, et al. . Toward automatic measurement of the linewith enhancement factor of semiconductor lasers using optical feedback self-mixing interferometry[J]. IEEE J. Quantum Elect. ,2007,43(7) :527-534.

[40] YU Y, XI J, CHICHARO J. Measuring the feedback parameter of a semiconductor laser with external optical feedback [J]. Optics Express,2011,19(107):9582-9593.

[41] FATHI M,DONATI S. Thickness measurement of transparent plates by a self-mix interferometer[J]. Opt. Lett. ,2010, $35: 1844-1846$

[42] FATHI M,DONATI S. Simultaneous measurement of thickness and refractive index by a single-channel self-mixing interferometer[J]. IET, Optoelectronics, 2011,6(1):7-12.

[43 ] DONATI S, ANNOVAZZI LODI V, MERLO S, et al. . Measurements of MEMS Mechanical parameters by Injection Interferometry [C]//Proc. IEEE-LEOS Internalional Conference on Optical MEMS ,Kauai, HI, USA Jan 21-24,2000:89-90.

[44] ANNOVAZZI LODI V, BENEDETTI M, MERLO S. Spot optical measurements on micromachined mirrors for photonics switching [J]. IEEE J. Quantum Elect. ,2004,10:536-544.

[45] DONATI S, NORGIA M, GIULIANI G. Self-mixing differential vibrometer based on electronic channel subtraction[J]. Appl. Optics, 2006, $45: 7264-7268$.

[46] DONATI S,SPEZIALI V. Laser interferometry for sensing of respiratory sounds[J]. IEEE J. Quantum Elect. ,1977,13 (9) :899-899.

[47] HAST J, MYLLYL R, SORVOJA H, et al. Arterial pulse shape measurement using self-mixing effect in a diode laser[J]. Quantum Electron,2002,32:975-982.

[48] ROVATI L, CATTINI S,PALANISAMY N. Measurement of the fluid-velocity profile using a self-mixing superluminescent diode[J]. Meas. Sci. Tech. ,2011,22:1-9.

[49] PESATORI A, NORGIA M,ROVATI L. Self-mixing laser doppler spectra of extracorporeal blood flow : A theoretical and experimental study $[\mathrm{J}]$. IEEE Sensor J. ,2011,12(3):552-557.

[50] KLIESE R, LIM Y L, BOSCH T, et al. . GaN laser self-mixing velocimeter for measuring slow flows [J]. Opt. Lett. , $2010,35: 814-816$.

[51] DONATI S,SOREL M. A phase-modulated feedback method for testing optical isolators assembled in the laser package [J]. IEEE Photonic Tech. Lett. ,1996,8: 405-408.

[52] DONATI S,SOREL M. High-sensitivity measurement of return loss by self-heterodyning in a laser diode[C]. Optical Fi- 
ber Communication Conference, OFC'97, Dallas, TX, USA, Feb 16-21,1997.

[53 ] LU C H, WANG J, DENG K L. Imaging and profiling surface microstructures with noninterferometric laser feedback [J]. Appl. Phys. Lett. ,1995,66:2022-2024.

[54] WANG M, LAI G. Self-mixing microscopic interferometer for the measurement of microprofile [J]. Optics Commun. , $2004,238: 237-244$.

作者简介：

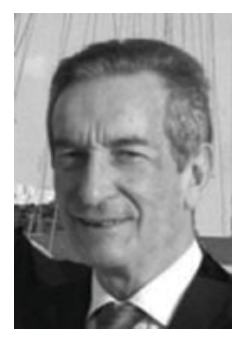

DONATI Silvano(1942-), 男, 意大利帕维亚人, 博士, 教授; IEEE 终身会士, OSA 会士, AEIT 功勋会员。在电子学 (CCD 中的噪音、耦合振荡器) 和光电仪器 (激光干涉仪、光纤 陀螺仪、光纤电流传感器) 等领域做出了突出贡献; 主要从事全光纤无源器件、光电探测器 中的噪音、光学混沌和密码术以及光混频等方面的研究。

E-mail:silvano.donati@ unipv. it

王 昭(1964一),女,陕西西安人,博士,教授,主要从事光电测量等方面的研究。

E-mail:wangzhao@mail.xjtu.edu.cn

禹延光 (1964一) 女, 河南郑州人, 博士, 高级讲师, 主要从事激光自混合干涉及数字图像处理等方面的研究。

E-mail:yanguang@uow.edu.au

\section{向您推荐《液晶与显示》期刊}

- 中文核心期刊

- 中国最早创办的液晶学科专业期刊之一

-中国液晶学科和显示技术领域中综合性学术期刊

- 中国物理学会液晶分会会刊, 中国光学光电子行业协会液晶分会会刊

- 英国《科学文摘》(SA)、美国《化学文摘》(CA)、俄罗斯《文摘杂志》(PW)、美国《剑桥科学文摘》 (CSA)、“中国科技论文统计源期刊”等 20 余种国内外著名检索刊物和文献数据库来源期刊

《液晶与显示》由中国科学院长春光学精密机械与物理研究所、中国物理学会液晶分会和中国光学 光电子行业协会液晶专业分会主办,科学出版社出版。

《液晶与显示》以研究报告、研究快报和综合评述等栏目集中报道国内外液晶学科和显示技术领域 中最新理论研究、科研成果和创新技术, 及时反映国内外本学科领域及产业信息动态, 内容丰富, 涵盖面 广, 信息量大, 可读性强, 是我国专业期刊发行量最大的刊物之一。

《液晶与显示》为双月刊, 国内定价 40.00 元。国内邮发代号: $12-203$; 国外发行代号:4868BM。

地 址: 长春市东南湖大路 3888 号

《液晶与显示》编辑部

邮 编: 130033

E-mail:yjxs@ ciomp.ac.cn
国内统一刊号:CN 22-1259/04

国际标准刊号:ISSN 1007-2780

电话: (0431) 6176059

网 址: www. yjyxs. com 